Elsevier required licence: (C) <2018>. This manuscript version is made available under the CC-BY-NC-ND 4.0 license http://creativecommons.org/licenses/by-nc-nd/4.0/ 


\section{An improved parametric level set method for structural frequency response optimization problems}

$\mathrm{LiHao}^{\mathrm{a}, \mathrm{b}}$

LuoZhen ${ }^{\mathrm{b}, *}$

zhen.luo@uts.edu.au

GaoLiang ${ }^{\mathrm{a}, *}$

gaoliang@mail.hust.edu.cn

WuJinglai ${ }^{a}$

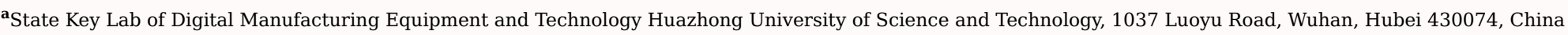

bSchool of Electrical, Mechanical and Mechatronic Systems The University of Technology, Sydney, 15 Broadway, Ultimo, NSW 2007, Australia

${ }^{*}$ Corresponding authors.

Abstract

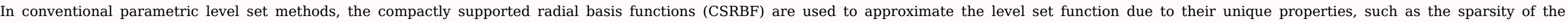

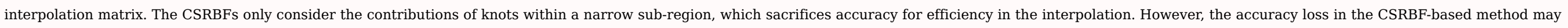

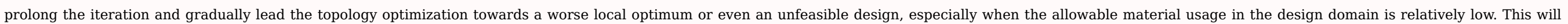

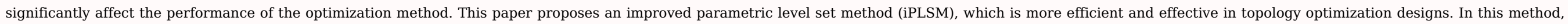

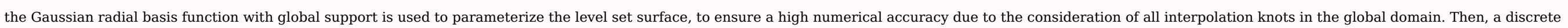

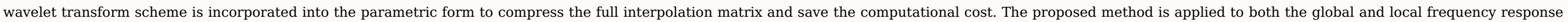
optimization problems under wide excitation frequency ranges, to validate its efficiency and effectiveness.

Keywords: Topology optimization; Level set method; Gaussian radial basis function; Discrete wavelet transform; Frequency response optimization

\section{Introduction}

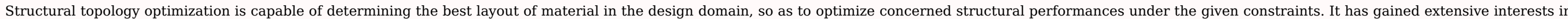

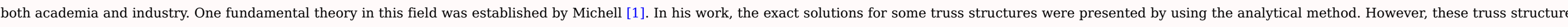

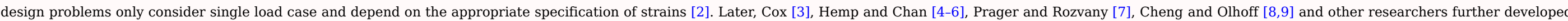

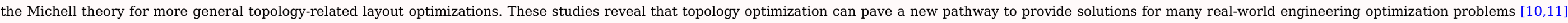

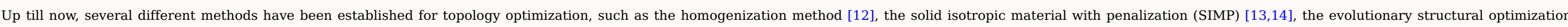
(ESO) method [15], as well as the level set method (LSM) [16-20], which are applied to a broad range of structure and material design problems [11,21].

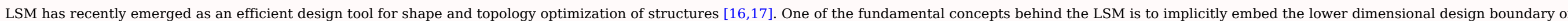

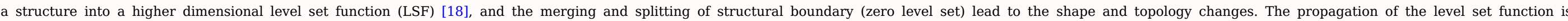

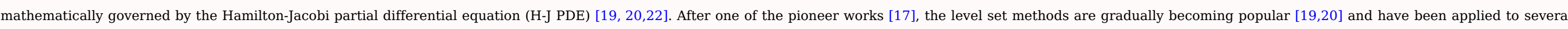

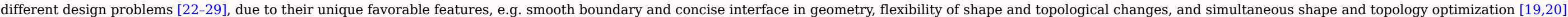




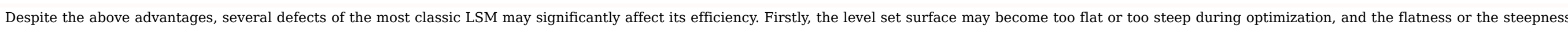

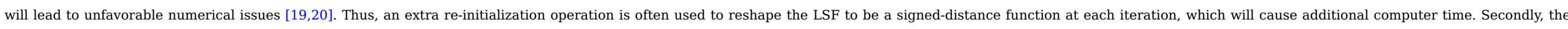

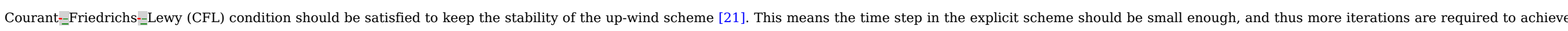

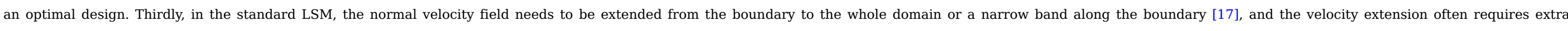
computational effort. More importantly, many efficient and well-developed structural topology optimization algorithms [13,30] cannot be directly used in the conventional LSM.

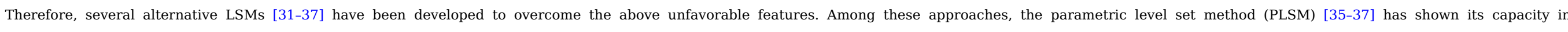

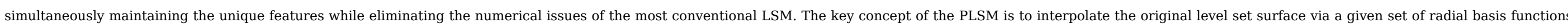

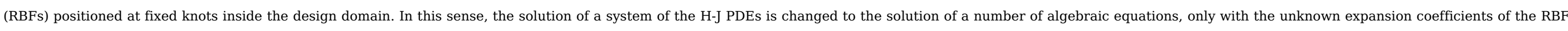

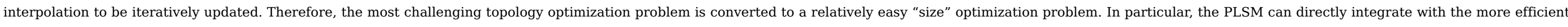
gradient-based optimization algorithms, such as the optimality criteria (OC) [13] and mathematical programming methods [30], which can't be easily applied to the conventional LSMs.

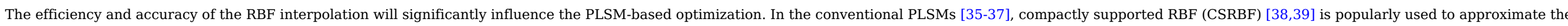

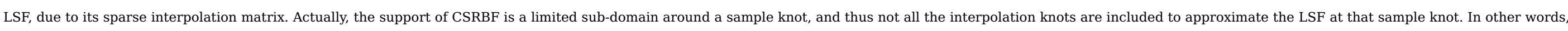

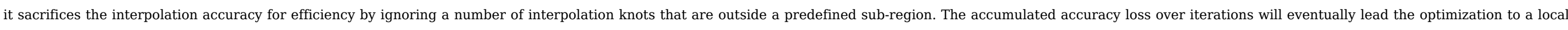

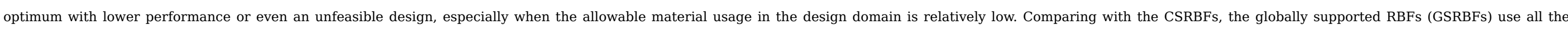

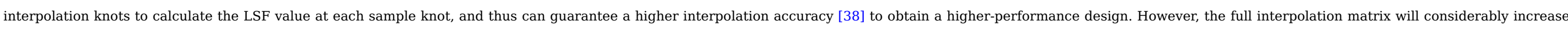
the computation effort. Indeed, a compressed interpolation matrix will be a reasonable solution to this computational difficulty.

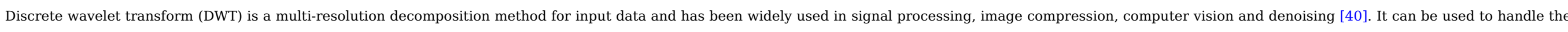

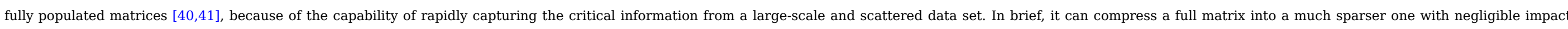

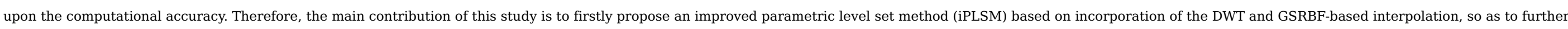
improve the efficiency and effectiveness of topological shape optimization.

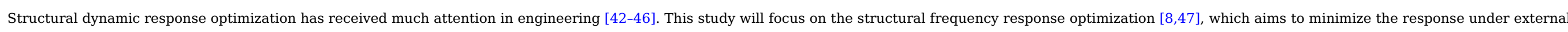

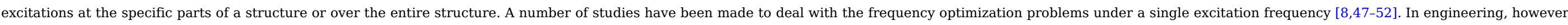

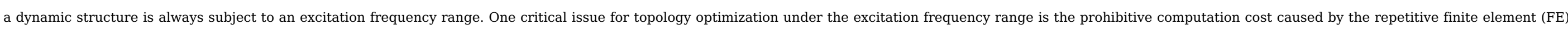

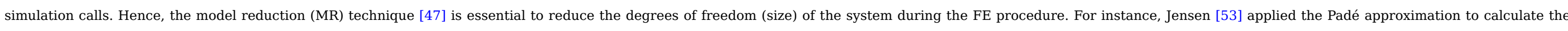

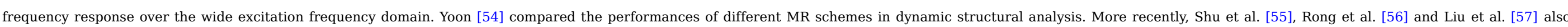

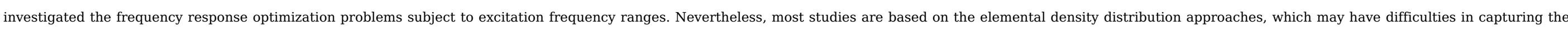

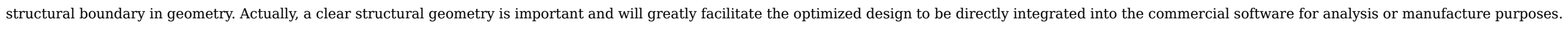

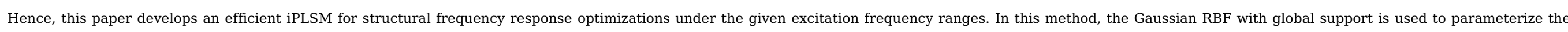

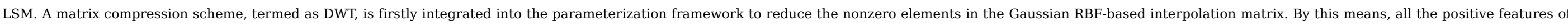

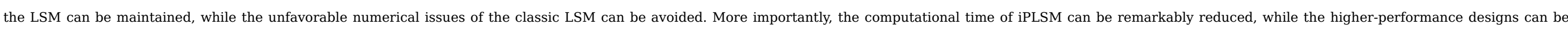

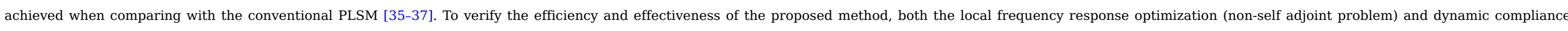
optimization (self adjoint problem) within wide excitation frequency ranges are investigated.

\section{Improved parametric level set method}

\subsection{Boundary representation and geometry mapping by LSM}

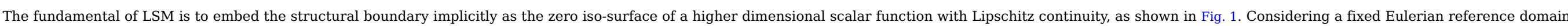




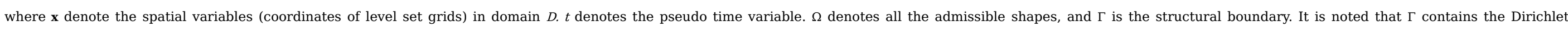
boundary $\Gamma_{D}$, Neumann boundary $\Gamma_{N}$ and traction free boundary $\Gamma_{F}$ :

$\Gamma=\Gamma_{D} \cup \Gamma_{N} \cup \Gamma_{F}$

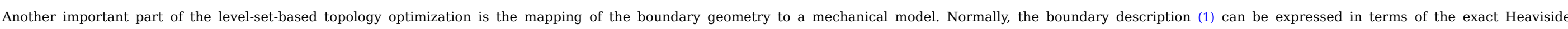
function $H(\Phi)$, so as to formulate integrals of some functional $J$ over the material domain [17,19-21]:

$\int_{\Omega} J d V=\int_{D} J \cdot H(\Phi) d V$

where

$H(\Phi)=\left\{\begin{array}{c}1, \Phi \geq 0 \\ 0, \Phi<0\end{array}\right.$

and $d V$ indicates a volume integral.

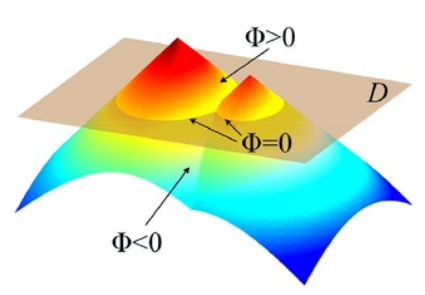

(a)

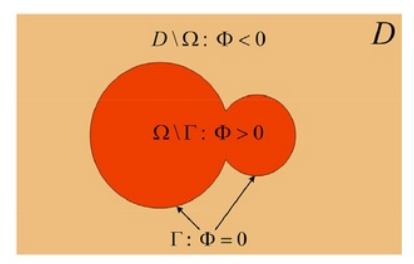

(b)

Fig. 1 (a) 3D level set surface and (b) 2D design domain.

\section{alt-text: Fig. 1.}

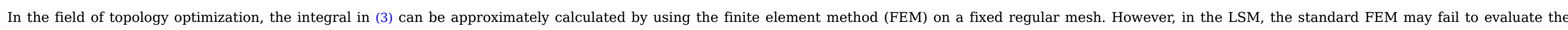

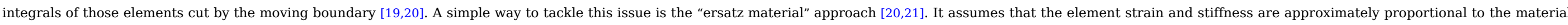
fraction of that element (i.e. pseudo element density), while the void elements are filled with weak material to avoid singularity. Thus, the volume integral of functional $J$ can be approximated by the FEM:

$\int_{D} J \cdot H(\Phi) d V \approx \int_{D} J \cdot \widetilde{H}(\Phi) d V \approx \sum_{h=1}^{N E} \chi_{h}(\Phi) \int_{D_{h}} J d V, h=1,2, \ldots, N E$

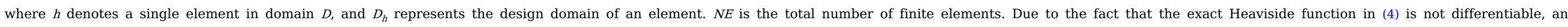
approximate Heaviside function $\widetilde{H}(\Phi)$ (smooth and differentiable) can be used to replace $H(\Phi)$ during topology optimization [21]:

$\widetilde{H}(\Phi)=\frac{1}{2}\left(1+\frac{2}{\pi} \arctan \left(\frac{\Phi}{\gamma}\right)\right)$

and the approximate Dirac delta function is given by:

$\tilde{\delta}(\Phi)=\frac{\partial \widetilde{H}(\Phi)}{\partial \Phi}=\frac{1}{\pi} \cdot \frac{\gamma}{\Phi^{2}+\gamma^{2}}$

where $\gamma$ is a small positive constant that is equal to $2 \cdot \Delta$ [36]. In this study, $\Delta$ denotes the edge length of a fixed regular level set grid. Then, the pseudo element density can be defined by: 
$\chi_{h}(\Phi)=\tau+(1-\tau) \frac{\int_{D_{h}} \widetilde{H}(\Phi) d V}{\int_{D_{h}} d V}$

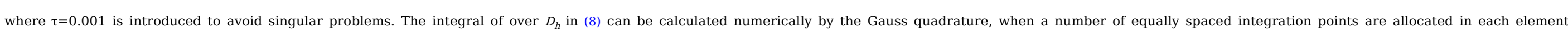
cut by the moving boundary [21]. In this case, the following integral can be also approximately calculated by FEM:

$\int_{D} J \cdot \tilde{\delta}(\Phi) d V \approx \sum_{h=1}^{N E} \frac{\partial \chi_{h}(\Phi)}{\partial \Phi} \cdot \int_{D_{h}} J d V=\sum_{h=1}^{N E}(1-\tau) \frac{\int_{D_{h}} \tilde{\delta}(\Phi) d V}{\int_{D_{h}} d V} \cdot \int_{D_{h}} J d V, h=1,2, \ldots, N E$

\subsection{Parameterization of LSM using Gaussian RBF}

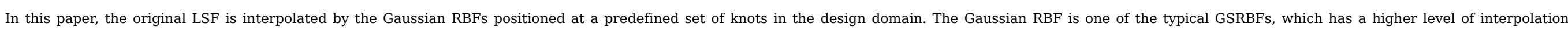
accuracy and smoothness when comparing with other RBFs, such as CSRBFs [38,39]. The Gaussian RBFs $\phi_{i}$ are expressed as:

$\varphi_{i}(\mathbf{x})=\mathrm{e}^{-s r_{i}(\mathbf{x})^{2}} \quad(i=1,2, \ldots, N)$

where $r$ defined in a Euclidean space can be stated by

$r_{i}(\mathbf{x})=\left\|\mathbf{x}-\mathbf{x}_{i}\right\|$

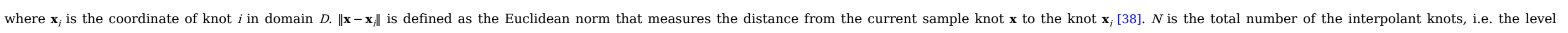
set knots in this study. $s$ is the shape parameter that is assumed to be a positive constant [38]. In this study, $s$ is equal to the reciprocal of the area (or volume) of a level set grid.

Using the Gaussian RBFs, the LSF is approximated by:

$\Phi(\mathbf{x}, t)=\varphi(\mathbf{x}) \alpha(t)=\sum_{i=1}^{N} \varphi_{i}(\mathbf{x}) \cdot \alpha_{i}(t)$

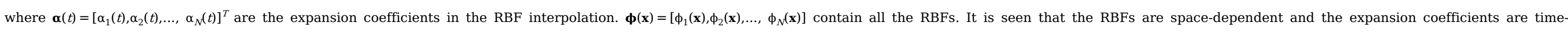

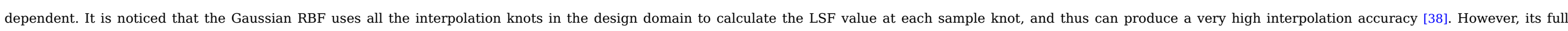
interpolation matrix will cause a remarkable computation burden during optimization. Using a matrix form, Eq. (12) can be rewritten as:

$\Phi=\mathbf{A} \alpha$, where $\Phi=\left[\Phi\left(\mathbf{x}_{1}, t\right), \Phi\left(\mathbf{x}_{2}, t\right), \ldots, \Phi\left(\mathbf{x}_{N}, t\right)\right]^{T}$

$\mathbf{A}=\left[\begin{array}{c}\varphi\left(\mathbf{x}_{1}\right) \\ \varphi\left(\mathbf{x}_{2}\right) \\ \ldots \\ \varphi\left(\mathbf{x}_{N}\right)\end{array}\right]=\left[\begin{array}{cccc}\varphi_{1}\left(\mathbf{x}_{1}\right) & \varphi_{2}\left(\mathbf{x}_{1}\right) & \ldots & \varphi_{N}\left(\mathbf{x}_{1}\right) \\ \varphi_{1}\left(\mathbf{x}_{2}\right) & \varphi_{2}\left(\mathbf{x}_{2}\right) & \ldots & \varphi_{N}\left(\mathbf{x}_{2}\right) \\ \ldots & \ldots & \ldots & \ldots \\ \varphi_{1}\left(\mathbf{x}_{N}\right) & \varphi_{2}\left(\mathbf{x}_{N}\right) & \ldots & \varphi_{N}\left(\mathbf{x}_{N}\right)\end{array}\right]$

Matrix $\mathbf{A}$ is invertible. The sparsity of $\mathbf{A}$ significantly influences the efficiency of the proposed method.

Substituting Eq. (12) into the H-J PDE [19], it yields a system of ordinary differential equations (ODEs):

$\varphi(\mathbf{x}) \frac{\partial \alpha(t)}{\partial t}-\vartheta_{\mathrm{n}}|\nabla(\varphi(\mathbf{x}) \alpha(t))|=0, \alpha(t=0)=\alpha_{0}$

where $\boldsymbol{\alpha}_{0}$ is the expansion coefficient vector at $t=0$, which is obtained by solving (13) with the initial level set function. Then the normal velocity field $\vartheta_{\mathrm{n}}$ is given by:

$\vartheta_{\mathrm{n}}=\frac{\varphi(\mathbf{x}) \alpha^{\prime}(t)}{|\nabla(\varphi(\mathbf{x}) \alpha(t))|}$, where $\alpha^{\prime}(t)=\frac{\partial \alpha(t)}{\partial t}$

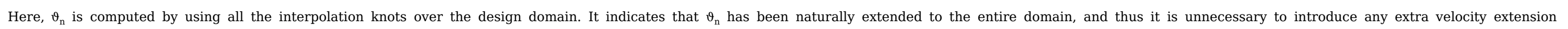




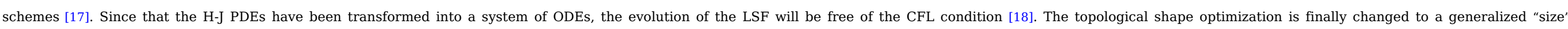
optimization, to efficiently update the expansion coefficients $\alpha_{i}(t)$ via the gradient-based optimization algorithms $[13,30]$.

\subsection{DWT-based interpolation matrix compression}

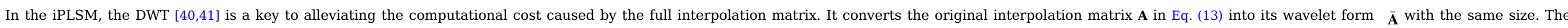

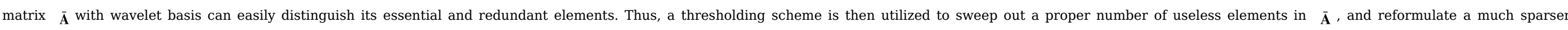
interpolation matrix $\widetilde{\mathbf{A}}_{s}$. Finally, the LSF in Eq. (13) can be efficiently evaluated by using the sparse matrix $\widetilde{\mathbf{A}}_{s}$.

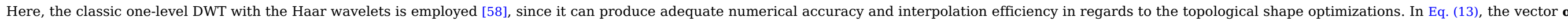
with length $N$ can be transformed into its wavelet basis by a pyramidal scheme [58]:

$\alpha_{k}^{(1)}=h_{1} \alpha_{2 k-1}^{(0)}+h_{2} \alpha_{2 k}^{(0)} ; d_{k}^{(1)}=g_{1} \alpha_{2 k-1}^{(0)}+g_{2} \alpha_{2 k}^{(0)}$

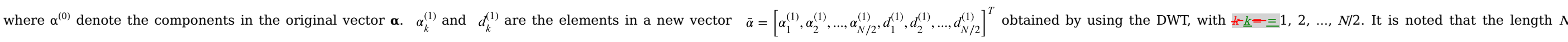

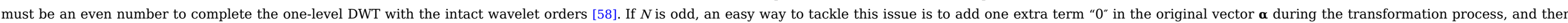

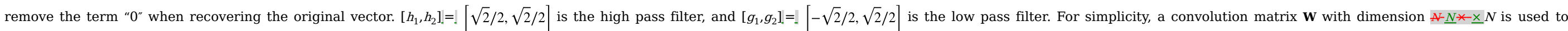
describe the pyramidal scheme [41]:

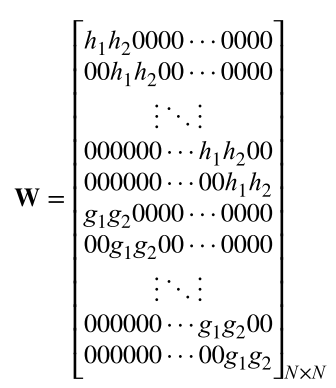

Using the orthogonal matrix $\mathbf{W}$, the level set function $\bar{\Phi}$, expansion coefficient $\bar{\alpha}$ and interpolation matrix $\overline{\mathbf{A}}$ after transformation can be respectively obtained by: $\bar{\Phi}=\mathbf{W} \Phi$ and $\bar{\alpha}=\mathbf{W} \alpha$

$\overline{\mathbf{A}}=\mathbf{W A W}^{T}$

With the pre-multiplication of $\mathbf{W}$ on both sides of (13), the interpolation process can be reformulated as:

$\mathbf{W} \Phi=\mathbf{W A}\left(\mathbf{W}^{T} \mathbf{W}\right) \alpha$

where the product of $\mathbf{W}^{T}$ and $\mathbf{W}$ yields a unit matrix.

Comparing the terms in Eqs. (19)-(21), one can obtain a new system of interpolation:

$\bar{\Phi}=\overline{\mathbf{A}} \bar{\alpha}$

Further, a sparse form of $\overline{\mathbf{A}}$, i.e. $\widetilde{\mathbf{A}}_{s}$, can be created by using the following threshold scheme [40]:

threshold $(q)=\left\{\begin{array}{l}q,|q| \geq \kappa \bar{q} ; \\ 0, \text { otherwise. }\end{array}\right.$

where $q$ denotes an arbitrary element in $\overline{\mathbf{A}}$, and $\bar{q}$ is the averaging absolute value of all elements in $\overline{\mathbf{A}} \cdot \mathrm{K}$ is a parameter to adjust the threshold. Now, a sparse form of (22) can be reformulated as:

$\bar{\Phi} \approx \widetilde{\mathbf{A}}_{s} \bar{\alpha}$

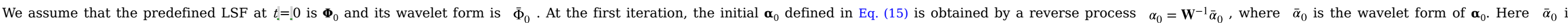




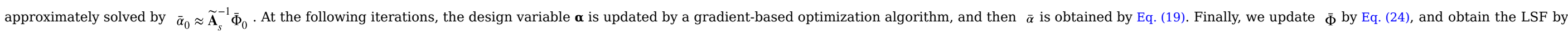
a reconstruction process $\Phi=\mathbf{W}^{-1} \bar{\Phi}$

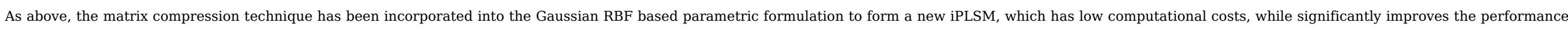

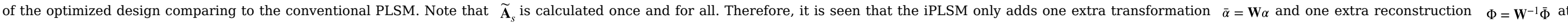
each iteration. Both the transformation and reconstruction use an extremely sparse system, whose computer cost is almost negligible.

\section{Frequency response topology optimization}

\subsection{Minimizing local frequency response}

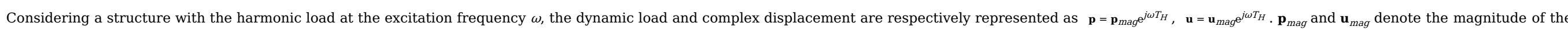

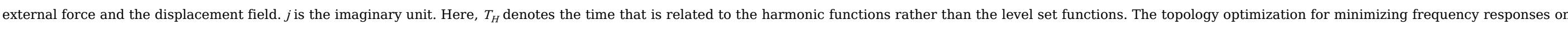
some given parts of a structure can be given by:

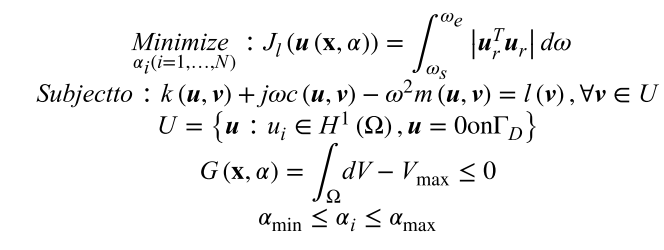

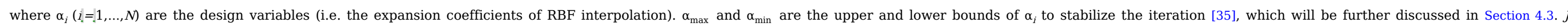

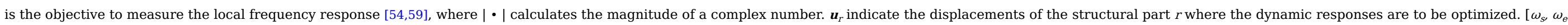

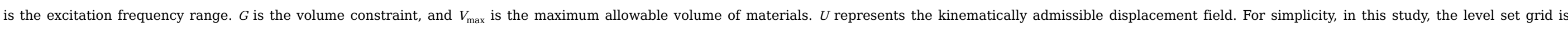
assumed to be identical with the FE mesh. Thus, $u_{i}$ is the displacement of an arbitrary FE (or level set) knot $i$ in domain $D$. $H^{1}(\Omega)$ is the first Sobolev function space.

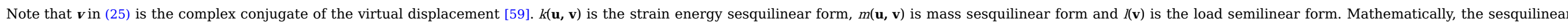

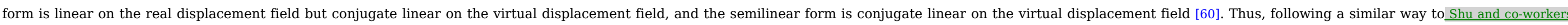
$[55,59,60]$, the following weak forms can be defined:

$$
\begin{aligned}
& k(\mathbf{u}, \mathbf{v})=\int_{\Omega} E_{p q r s} \varepsilon_{p q}(\mathbf{u}) \varepsilon_{r s}(\mathbf{v}) d V \\
& m(\mathbf{u}, \mathbf{v})=\int_{\Omega} \rho \mathbf{u v} d V \\
& l(\mathbf{v})=\int_{\Gamma_{N}} \mathbf{p v} d S
\end{aligned}
$$

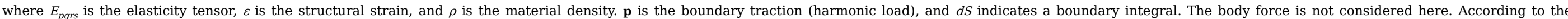
Rayleigh damping assumption, the damping functional in (25) is calculated by the linear combination of the strain energy and mass sesquilinear forms with constants $\beta_{1}$ and $\beta_{2}$ [61]

$c(\mathbf{u}, \mathbf{v})=\beta_{1} k(\mathbf{u}, \mathbf{v})+\beta_{2} m(\mathbf{u}, \mathbf{v})$

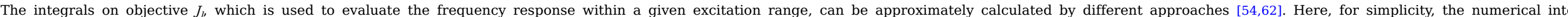
scheme with equally spaced abscissas [54] is adopted. We denote the local frequency response at a specified excitation frequency $\omega_{z} \in\left[\omega_{s}, \omega_{e}\right]$ as

$f_{l}^{\omega_{z}}(\mathbf{u})$, where $\omega_{z}=\omega_{s}+z \cdot\left(\omega_{e}-\omega_{s}\right) / N F$

$N F=\left(\omega_{e}-\omega_{s}\right) / \Delta \omega$

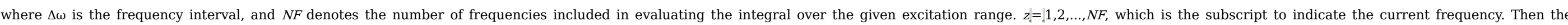


$J_{l}(\mathbf{u}) \approx \frac{\Delta \omega}{2} \cdot J_{l}^{\omega_{1}}(\mathbf{u})+\sum_{z=2}^{N F-1} \Delta \omega \cdot J_{l}^{\omega_{z}}(\mathbf{u})+\frac{\Delta \omega}{2} \cdot f_{l}^{\omega_{N F}}(\mathbf{u})$

\subsection{Minimizing dynamic compliance}

The topology optimization model for minimizing the global frequency response is formulated as:

$\underset{\alpha_{i}(i=1, \ldots, N)}{\operatorname{Minimize}}: J_{g}(\boldsymbol{u}(\mathbf{x}, \alpha))=\int_{\omega_{S}}^{\omega_{e}}\left|\int_{\Omega} \boldsymbol{p} \boldsymbol{u} d V\right| d \omega$

Subjectto : $k(\boldsymbol{u}, \boldsymbol{v})+j \omega c(\boldsymbol{u}, \boldsymbol{v})-\omega^{2} m(\boldsymbol{u}, \boldsymbol{v})=l(\boldsymbol{v}), \forall \boldsymbol{v} \in U$

$k(\boldsymbol{u}, \boldsymbol{v})+j \omega c(\boldsymbol{u}, \boldsymbol{v})-\omega^{2} m(\boldsymbol{u}, \boldsymbol{v})=l$
$U=\left\{\boldsymbol{u}: u_{i} \in H^{1}(\Omega), \boldsymbol{u}=0\right.$ on $\left.\Gamma_{D}\right\}$

$G(\mathbf{x}, \alpha)=\int_{\Omega} d V-V_{\max } \leq 0$

$$
\alpha_{\min } \leq \alpha_{i} \leq \alpha_{\max }
$$

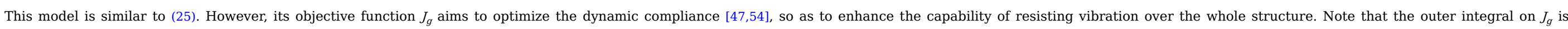
also calculated by a similar scheme given in (32).

\section{Sensitivity analysis and optimization algorithm}

\subsection{Design sensitivity analysis for minimizing local frequency response}

Based on Eq. (32), it can be seen that the objective $J_{l}$ is evaluated by $J_{l}^{\omega_{z}}$. Thus, it is essential to derive the sensitivity information for $J_{l}^{\omega_{z}}$ :

$J_{l}^{\omega_{z}}=\left|\mathbf{u}_{r}^{T} \mathbf{u}_{r}\right|=\left|J_{u}\right|=\sqrt{\left(\operatorname{real}\left(J_{u}\right)\right)^{2}+\left(\operatorname{imag}\left(J_{u}\right)\right)^{2}}$

where $J_{u}$ is introduced to denote $\mathbf{u}_{r}^{T} \mathbf{u}_{r} \cdot \operatorname{real}(\bullet)$ and imagl $\bullet$ ) respectively acquire the real and imaginary parts of a functional. According to Ref. [59], the design sensitivity of $J_{l}^{\omega_{z}}$ is derived by:

$\frac{\partial f_{l}^{\omega_{z}}}{\partial \alpha_{i}}=\left(\operatorname{real}\left(J_{u}\right) \cdot \operatorname{real}\left(\frac{\partial J_{u}}{\partial \alpha_{i}}\right)+\operatorname{imag}\left(J_{u}\right) \cdot \operatorname{imag}\left(\frac{\partial J_{u}}{\partial \alpha_{i}}\right)\right) / \sqrt{\left(\operatorname{real}\left(J_{u}\right)\right)^{2}+\left(\operatorname{imag}\left(J_{u}\right)\right)^{2}}$

In Eq. (35), $J_{u}$ can be obtained by FEM. Thus, we can now focus on the calculation of $\partial J_{u} / \partial \alpha_{i}$. To this end, we firstly present the derivative $\partial J_{u} / \partial t$ :

$\frac{\partial J_{u}}{\partial t}=\sum_{i=1}^{N} \frac{\partial J_{u}}{\partial \alpha_{i}(t)} \alpha_{i}^{\prime}(t)=2 \mathbf{u}_{r}^{T} \mathbf{u}_{r}^{\prime}$

where $\mathbf{u}_{r}^{\prime}$ denotes the derivative of $\mathbf{u}_{r}$ with respect to $t$

The shape derivative $[60,63]$ of the state equation in Eq. (25) can be derived as:

$$
\begin{gathered}
\left(1+j \omega \beta_{1}\right)\left[k\left(\boldsymbol{u}^{\prime}, \boldsymbol{v}\right)+k\left(\boldsymbol{u}, \boldsymbol{v}^{\prime}\right)+\int_{\Gamma} E_{p q r s} \varepsilon_{p q}(\mathbf{u}) \varepsilon_{r s}(\mathbf{v}) \vartheta_{\mathrm{n}} d S\right] \\
+\left(j \omega \beta_{2}-\omega^{2}\right)\left[m\left(\boldsymbol{u}^{\prime}, \boldsymbol{v}\right)+m\left(\boldsymbol{u}, \boldsymbol{v}^{\prime}\right)+\int_{\Gamma} \rho \mathbf{u v} \vartheta_{\mathrm{n}} d S\right] \\
=\int_{\Gamma_{N}} \boldsymbol{p} \boldsymbol{v}^{\prime} d S+\int_{\Gamma_{N}}(\nabla(\boldsymbol{p} \boldsymbol{v}) \cdot \mathbf{n}+(\nabla \cdot \mathbf{n}) \cdot(\boldsymbol{p} \boldsymbol{v})) \vartheta_{\mathrm{n}} d S
\end{gathered}
$$

where $\mathbf{u}^{\prime}$ and $\mathbf{v}^{\prime}$ represent the derivatives of $\mathbf{u}$ and $\mathbf{v}$ with respect to $t . \mathbf{n}$ is the outward normal vector along the boundary of $\Omega[19,20]$

Considering that $\mathbf{v}^{\prime} \in U$, we have the following vibrational state equation:

$\left(1+j \omega \beta_{1}\right) k\left(\boldsymbol{u}, \boldsymbol{v}^{\prime}\right)+\left(j \omega \beta_{2}-\omega^{2}\right) m\left(\boldsymbol{u}, \boldsymbol{v}^{\prime}\right)=\int_{\Gamma_{N}} \boldsymbol{p} \boldsymbol{v}^{\prime} d S$ 


$$
\begin{gathered}
\left(1+j \omega \beta_{1}\right) k\left(\boldsymbol{u}^{\prime}, \boldsymbol{v}\right)+\left(j \omega \beta_{2}-\omega^{2}\right) m\left(\boldsymbol{u}^{\prime}, \boldsymbol{v}\right)=\int_{\Gamma_{N}}(\nabla(\boldsymbol{p} v) \cdot \mathbf{n}+(\nabla \cdot \mathbf{n}) \cdot(\boldsymbol{p} v)) \vartheta_{\mathrm{n}} d S \\
-\int_{\Gamma}\left(\left(1+j \omega \beta_{1}\right) E_{p q r s} \varepsilon_{p q}(\boldsymbol{u}) \varepsilon_{r s}(\boldsymbol{v})+\left(j \omega \beta_{2}-\omega^{2}\right) \rho \mathbf{u v}\right) \vartheta_{\mathrm{n}} d S
\end{gathered}
$$

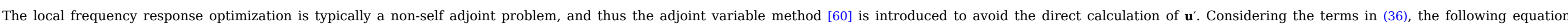
can be derived from (39) by replacing $\mathbf{v}$ with the adjoint variable $\mathbf{w}$

$$
\begin{gathered}
2 \mathbf{u}_{r}^{T} \mathbf{u}_{r}^{\prime}=\int_{\Gamma_{N}}(\nabla(\mathbf{p w}) \cdot \mathbf{n}+(\nabla \cdot \mathbf{n}) \cdot(\mathbf{p w})) \vartheta_{\mathrm{n}} d S \\
-\int_{\Gamma}\left(\left(1+j \omega \beta_{1}\right) E_{p q r s} \varepsilon_{p q}(\mathbf{u}) \varepsilon_{r s}(\mathbf{w})+\left(j \omega \beta_{2}-\omega^{2}\right) \rho \mathbf{u w}\right) \vartheta_{\mathrm{n}} d S
\end{gathered}
$$

where $\mathbf{w}$ is equal to the complex conjugate of variable $\mathbf{w}^{*}[60] . \mathbf{w}^{*}$ can be obtained by solving the adjoint Eq. (41), which has the same sesquilinear form of the state equation in Eq. (25): $k\left(\overline{\mathbf{w}}, \mathbf{w}^{*}\right)+j \omega c\left(\overline{\mathbf{w}}, \mathbf{w}^{*}\right)-\omega^{2} m\left(\overline{\mathbf{w}}, \mathbf{w}^{*}\right)=l_{a}(\overline{\mathbf{w}}), \forall \overline{\mathbf{w}} \in U$

where $\overline{\mathbf{w}}$ is the virtual adjoint variable, and $l_{a}(\overline{\mathbf{w}})=2 \mathbf{u}_{r}^{T}$ is regarded as the adjoint load term [55,60].

Comparing the terms in (36) and (40), the shape derivative of $J_{u}$ is obtained by:

$\frac{\partial J_{u}}{\partial t}=-\int_{\Gamma}\left(\left(1+j \omega \beta_{1}\right) E_{p q r s} \varepsilon_{p q}(\mathbf{u}) \varepsilon_{r s}(\mathbf{w})+\left(j \omega \beta_{2}-\omega^{2}\right) \rho \mathbf{u w}\right) \vartheta_{\mathrm{n}} d S$

It should be noted that $\Gamma_{N}$ is assumed to be fixed while (42) is being derived, and thus the integral over $\Gamma_{N}$ vanishes. Substituting $\vartheta_{\mathrm{n}}$ defined in (16) into (42), it yields:

$\frac{\partial J_{u}}{\partial t}=-\int_{\Gamma}\left(\left(1+j \omega \beta_{1}\right) E_{p q r s} \varepsilon_{p q}(\mathbf{u}) \varepsilon_{r s}(\mathbf{w})+\left(j \omega \beta_{2}-\omega^{2}\right) \rho \mathbf{u w}\right) \frac{\varphi(\mathbf{x}) \alpha^{\prime}(t)}{|\nabla(\varphi(\mathbf{x}) \alpha(t))|} d S$

When introducing the Dirac delta function given in (7), we can have following mapping relation [21]:

$\int_{\Gamma} d S=\int_{D} \tilde{\delta}(\Phi)|\nabla \Phi| d V$

To facilitate the implementation, Eq. (43) can be reformulated as a volume integral via Eq. (44) [36]:

$\frac{\partial J_{u}}{\partial t}=-\int_{D}\left(\left(1+j \omega \beta_{1}\right) E_{p q r s} \varepsilon_{p q}(\mathbf{u}) \varepsilon_{r s}(\mathbf{w})+\left(j \omega \beta_{2}-\omega^{2}\right) \rho \mathbf{u w}\right) \tilde{\delta}(\Phi) \varphi(\mathbf{x}) \alpha^{\prime}(t) d V$

$=-\sum_{i=1}^{N} \int_{D}\left(\left(1+j \omega \beta_{1}\right) E_{p q r s} \varepsilon_{p q}(\mathbf{u}) \varepsilon_{r s}(\mathbf{w})+\left(j \omega \beta_{2}-\omega^{2}\right) \rho \mathbf{u w}\right) \tilde{\delta}(\Phi) \varphi_{i}(\mathbf{x}) \alpha_{i}^{\prime}(t) d V$

Comparing the corresponding terms in (36) and (45), and eliminating $\alpha_{i}^{\prime}(t)$, it yields:

$\frac{\partial J_{u}}{\partial \alpha_{i}}=-\int_{D}\left(\left(1+j \omega \beta_{1}\right) E_{p q r s} \varepsilon_{p q}(\mathbf{u}) \varepsilon_{r s}(\mathbf{w})+\left(j \omega \beta_{2}-\omega^{2}\right) \rho \mathbf{u w}\right) \tilde{\delta}(\Phi) \varphi_{i}(\mathbf{x}) d V$

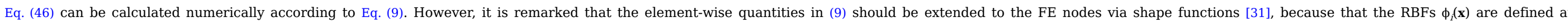

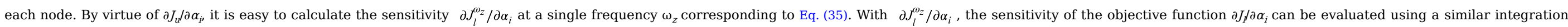
scheme given in (32).

Similarly, the sensitivity of the volume constraint can be derived as:

$\frac{\partial G}{\partial \alpha_{i}}=\int_{D} \tilde{\delta}(\Phi) \varphi_{i}(\mathbf{x}) d V$

\subsection{Design sensitivity analysis for minimizing dynamic compliance}

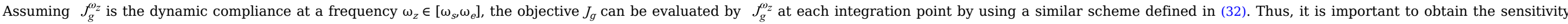
information for $f_{g}^{\omega_{z}}$ : 
$J_{g}^{o_{z}}=\left|\int_{\Omega} \mathbf{p u} d V\right|=\left|J_{d}\right|=\sqrt{\left(\operatorname{real}\left(J_{d}\right)\right)^{2}+\left(\operatorname{imag}\left(J_{d}\right)\right)^{2}}$

where $J_{d}$ is introduced to denote $\int_{\Omega} \boldsymbol{p} \boldsymbol{u} d V$. The design sensitivity of $f_{g}^{\omega_{z}}$ is then given by:

$\frac{\partial J_{g}^{\omega_{z}}}{\partial \alpha_{i}}=\left(\operatorname{real}\left(J_{d}\right) \cdot \operatorname{real}\left(\frac{\partial J_{d}}{\partial \alpha_{i}}\right)+\operatorname{imag}\left(J_{d}\right) \cdot \operatorname{imag}\left(\frac{\partial J_{d}}{\partial \alpha_{i}}\right)\right) / \sqrt{\left(\operatorname{real}\left(J_{d}\right)\right)^{2}+\left(\operatorname{imag}\left(J_{d}\right)\right)^{2}}$

$J_{d}$ is obtained by FEM, and we now focus on the calculation of $\partial J_{d} / \partial \alpha_{i}$. Here, $J_{d}$ is re-written by:

$$
\begin{gathered}
J_{d}=\int_{\Omega}\left(1+j \omega \beta_{1}\right) E_{p q r s} \varepsilon_{p q}(\boldsymbol{u}) \varepsilon_{r s}(\boldsymbol{u}) d V+\int_{\Omega}\left(j \omega \beta_{2}-\omega^{2}\right) \rho \mathbf{u u} d V \\
=\left(1+j \omega \beta_{1}\right) k(\boldsymbol{u}, \boldsymbol{u})+\left(j \omega \beta_{2}-\omega^{2}\right) m(\boldsymbol{u}, \boldsymbol{u})
\end{gathered}
$$

The shape derivative of $J_{d}$ can be derived by:

$$
\begin{aligned}
& \frac{\partial J_{d}}{\partial t}=2\left(1+j \omega \beta_{1}\right) k\left(\boldsymbol{u}^{\prime}, \boldsymbol{u}\right)+2\left(j \omega \beta_{2}-\omega^{2}\right) m\left(\boldsymbol{u}^{\prime}, \boldsymbol{u}\right) \\
& +\int_{\Gamma}\left(\left(1+j \omega \beta_{1}\right) E_{p q r s} \varepsilon_{p q}(\boldsymbol{u}) \varepsilon_{r s}(\boldsymbol{u})+\left(j \omega \beta_{2}-\omega^{2}\right) \rho \mathbf{u u}\right) \vartheta_{\mathrm{n}} d S
\end{aligned}
$$

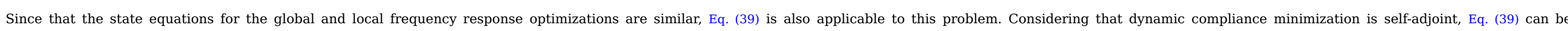
rewritten by:

$$
\begin{gathered}
\left(1+j \omega \beta_{1}\right) k\left(\boldsymbol{u}^{\prime}, \boldsymbol{u}\right)+\left(j \omega \beta_{2}-\omega^{2}\right) m\left(\boldsymbol{u}^{\prime}, \boldsymbol{u}\right)=\int_{\Gamma_{N}}(\nabla(\boldsymbol{p} \boldsymbol{u}) \cdot \mathbf{n}+(\nabla \cdot \mathbf{n}) \cdot(\boldsymbol{p} \boldsymbol{u})) \vartheta_{\mathrm{n}} d S \\
-\int_{\Gamma}\left(\left(1+j \omega \beta_{1}\right) E_{p q r s} \varepsilon_{p q}(\boldsymbol{u}) \varepsilon_{r s}(\boldsymbol{u})+\left(j \omega \beta_{2}-\omega^{2}\right) \rho \mathbf{u u}\right) \vartheta_{\mathrm{n}} d S
\end{gathered}
$$

Then the shape derivative of $J_{d}$ can be obtained by substituting Eq. (52) into Eq. (51):

$\frac{\partial J_{d}}{\partial t}=-\int_{\Gamma}\left(\left(1+j \omega \beta_{1}\right) E_{p q r s} \varepsilon_{p q}(\boldsymbol{u}) \varepsilon_{r s}(\boldsymbol{u})+\left(j \omega \beta_{2}-\omega^{2}\right) \rho \mathbf{u u}\right) \vartheta_{\mathrm{n}} d S$

Note that $\Gamma_{N}$ is fixed while (53) is being derived, and thus the integral over $\Gamma_{N}$ is eliminated.

Similar to the process described by Eqs. (43)-=(45), we can reformulate $\partial J_{d} / \partial t$ as:

$\frac{\partial J_{d}}{\partial t}=-\sum_{i=1}^{N} \int_{D}\left(\left(1+j \omega \beta_{1}\right) E_{p q r s} \varepsilon_{p q}(\mathbf{u}) \varepsilon_{r s}(\mathbf{u})+\left(j \omega \beta_{2}-\omega^{2}\right) \rho \mathbf{u u}\right) \tilde{\delta}(\Phi) \varphi_{i}(\mathbf{x}) \alpha^{\prime}{ }_{i}(t) d V$

On the other hand, $\partial J_{d} / \partial t$ can be also derived by the chain rule:

$\frac{\partial J_{d}}{\partial t}=\sum_{i=1}^{N} \frac{\partial J_{d}}{\partial \alpha_{i}(t)} \alpha_{i}^{\prime}(t)$

By comparing the corresponding terms in (54) and (55), $\partial J_{d} / \partial \alpha_{i}$ can be easily given by:

$\frac{\partial J_{d}}{\partial \alpha_{i}}=-\int_{D}\left(\left(1+j \omega \beta_{1}\right) E_{p q r s} \varepsilon_{p q}(\mathbf{u}) \varepsilon_{r s}(\mathbf{u})+\left(j \omega \beta_{2}-\omega^{2}\right) \rho \mathbf{u u}\right) \tilde{\delta}(\Phi) \varphi_{i}(\mathbf{x}) d V$

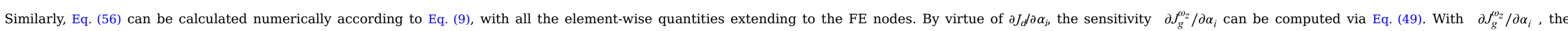
sensitivity of the objective function $\partial J_{g} / \partial \alpha_{i}$ can be calculated by a similar integration scheme defined in (32). The sensitivity of volume constraint is the same as (47).

\subsection{Optimization algorithm}

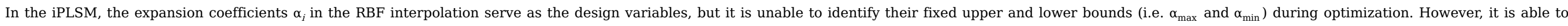
specify the fixed bounds $\tilde{\alpha}_{\min } \leq \widetilde{\alpha}_{i} \leq \widetilde{\alpha}_{\max }$ on the regularized design variables $\widetilde{\alpha}_{i}$. Hence, an OC-based optimization algorithm [10,13] can be established, as follows:

Step 1 Calculating the regularized design variables 
$\widetilde{\alpha}_{i}^{(\xi)}=\frac{\alpha_{i}^{(\xi)}-\alpha_{\min }^{(\xi)}}{\alpha_{\max }^{(\xi)}-\alpha_{\min }^{(\xi)}}$, where $\alpha_{\max }^{(\xi)}=2 \times \max \left(\alpha_{i}^{(\xi)}\right), \alpha_{\min }^{(\xi)}=2 \times \min \left(\alpha_{i}^{(\xi)}\right)$

where $\xi$ indicates the current iteration number.

Step 2 Based on the Kuhn-Tucker conditions [10,13], the following heuristic scheme can be established to iteratively update the regularized design variables $\widetilde{\alpha}_{i}$ :

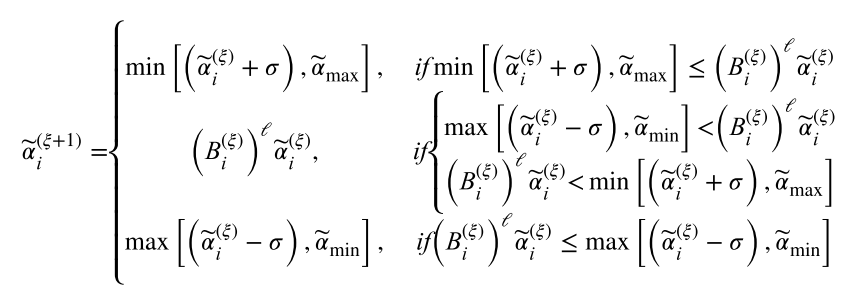

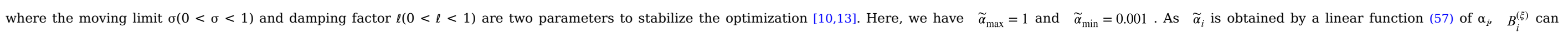
be given by:

$B_{i}^{(\xi)}=-\frac{\partial J_{l}}{\partial \alpha_{i}^{(\xi)}} / \max \left(\mu, \Lambda^{(\xi)} \frac{\partial G}{\partial \alpha_{i}^{(\xi)}}\right) o r-\frac{\partial J_{g}}{\partial \alpha_{i}^{(\xi)}} / \max \left(\mu, \Lambda^{(\xi)} \frac{\partial G}{\partial \alpha_{i}^{(\xi)}}\right)$

where $\mu=1 \mathrm{e}-10$ is used to avoid the zero terms. At each iteration, the Lagrange multipliers $\Lambda^{(\xi)}$ can be found by a bi-sectioning approach [10].

Step 3 Updating the design variables:

$\alpha_{i}^{(\xi+1)}=\widetilde{\alpha}_{i}^{(\xi+1)} \times\left(\alpha_{\max }^{(\xi)}-\alpha_{\min }^{(\xi)}\right)+\alpha_{\min }^{(\xi)}$

Step 4 Repeating Step 1 to Step 3 until a convergent criterion is reached.

\section{Numerical implementations}

\subsection{Localized modes}

In the most standard SIMP topology optimization [13,14], the element density $\rho_{h}^{S I M P}$ and Young's modulus $E_{h}^{S I M P}$ are interpolated by: $\rho_{h}^{S I M P}=\eta_{h} \rho, E_{h}^{S I M P}=\left(\eta_{h}\right)^{p} E$

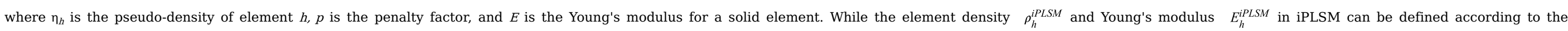
ersatz material model given in Eq. (5):

$\rho_{h}^{i P L S M}=\chi_{h} \rho, E_{h}^{i P L S M}=\chi_{h} E$

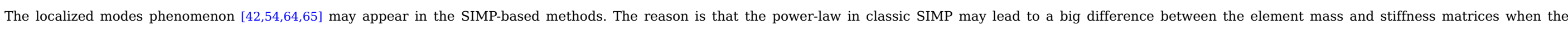

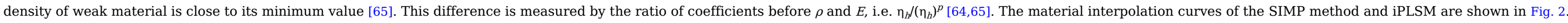
In the ersatz material model, the ratio of coefficients before $\rho$ and $E$ in iPLSM is always equivalent to 1 . In this case, the localized modes can be naturally avoided. 


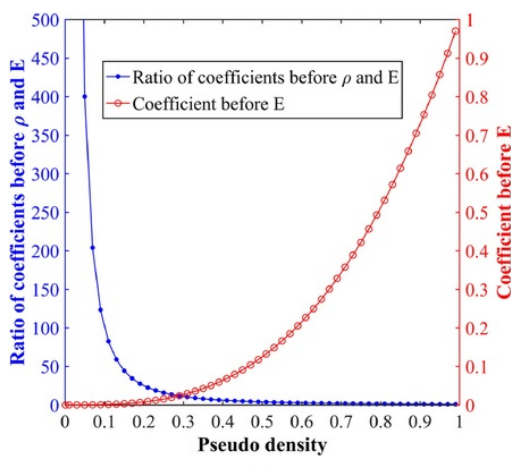

(a)

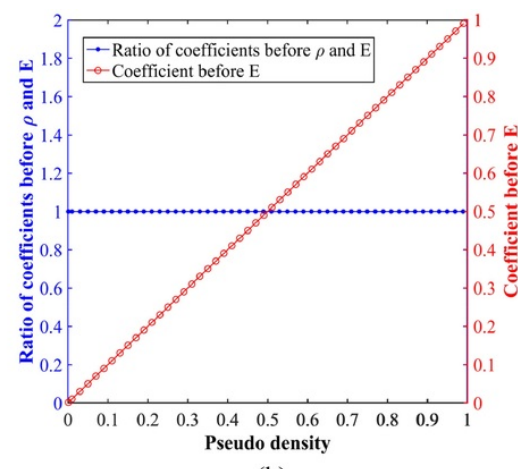

(b)

Fig. 2 Material interpolation curves: (a) classic SIMP method with $p=3$ and (b) iPLSM.

\section{alt-text: Fig. 2.}

\subsection{Filtering scheme}

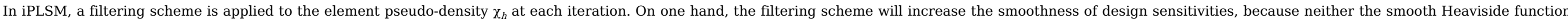

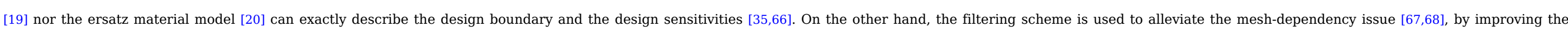
continuity of the pseudo-density field.

The filter on element pseudo-density $\chi_{h}$ can be defined as:

$$
\begin{aligned}
& \bar{\chi}_{h}=\sum_{h=1}^{N E}\left(\sum_{f=1}^{N H} \theta_{h,} \chi_{f} / \sum_{f=1}^{N H} \theta_{h, f}\right), \text { where }_{h_{h} f}=\bar{\theta}_{h, f} / \sum_{f=1}^{N H} \bar{\theta}_{h, f} \\
& \bar{\theta}_{h, f}=\sum_{h=1}^{N E}\left(\sum_{f=1}^{N H} \frac{3}{\pi r_{\min }^{2}} \max \left(0, \frac{r_{\min }-\operatorname{dist}(h, f)}{r_{\min }}\right)\right)
\end{aligned}
$$

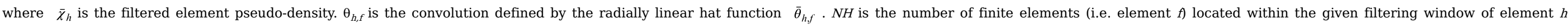
$r_{\min }$ defines the radius of the filter area, which is typically $1.5-2.0$ times of the mesh size. $\operatorname{dist}(h, f)$ represents the distance between element $h$ and $f$.

\subsection{Model reduction scheme}

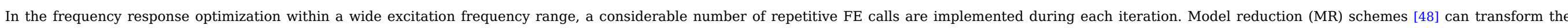

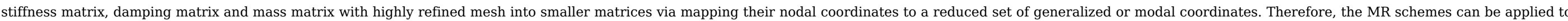

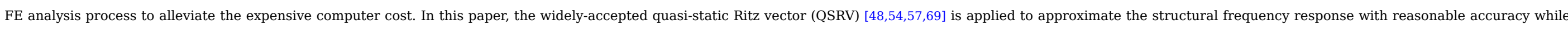
reduce the computational cost. The readers can refer to Ma and co-workers $[48,54,57,69]$ for more details about the well-established QSRV approach.

\section{Numerical examples}

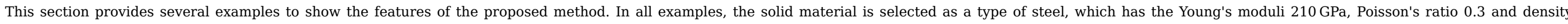

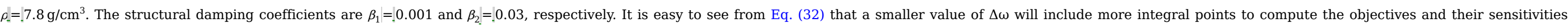

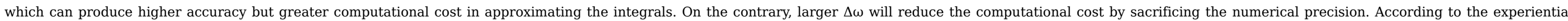

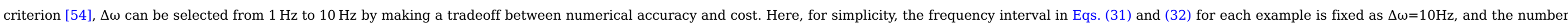

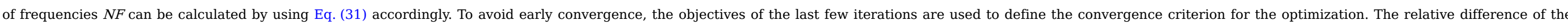
objective functions between two successive steps at the current iteration $\xi$ is denoted as $\Upsilon^{(\xi)}$. The iteration is terminated when $\Upsilon^{(\xi-2)}$, $\Upsilon^{(\xi-1)}$ and $\Upsilon^{(\xi)}$ are all less than 0.0005 . 


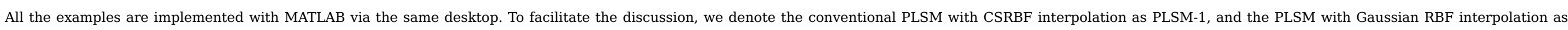

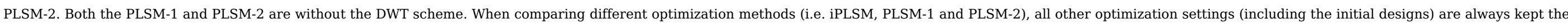
same except their interpolation mechanisms.

\subsection{Local frequency response optimization for excited point of 2D structure}

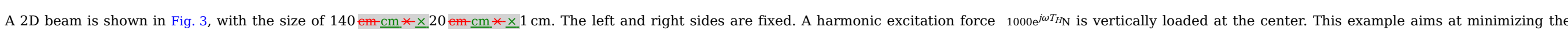
frequency response at the loaded point $\mathbf{P}$ with the excitation frequency range $\Omega_{\text {freq }}=[0 \mathrm{~Hz}, 100 \mathrm{~Hz}]$. The maximum material usage is restricted to $40 \%$.

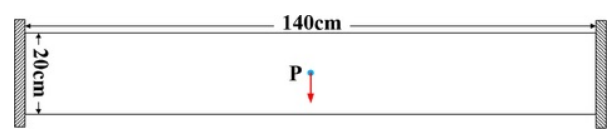

Fig. 3 Design domain of first example.

alt-text: Fig. 3.

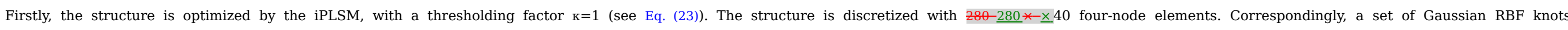

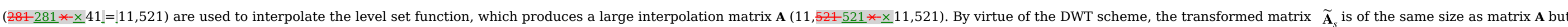

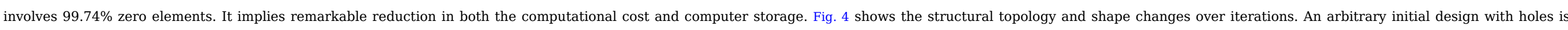

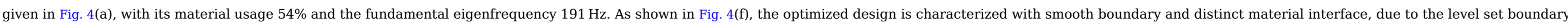

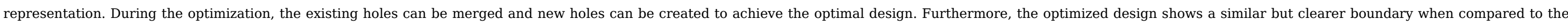
result given in [48].

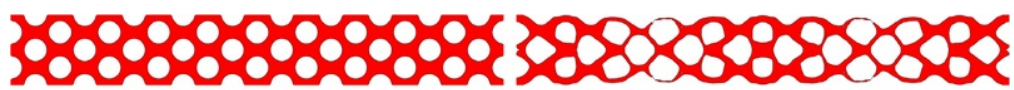

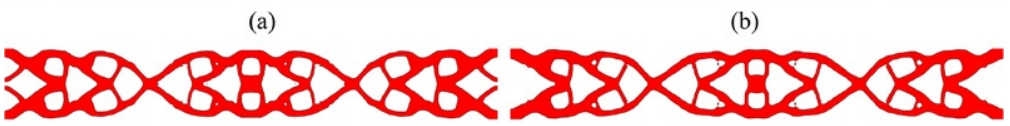 \\ (c)

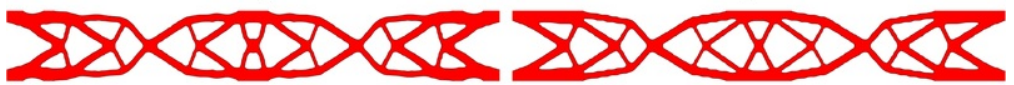

(e)

(f)

Fig. 4 Evolution of structural topology and shape: (a) initial design; (b) step 10; (c) step 15; (d) step 25; (e) step 50; (f) optimized design. alt-text: Fig. 4.

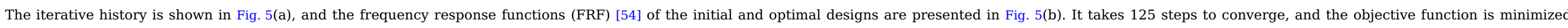

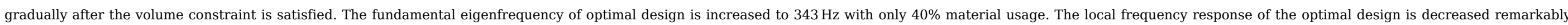

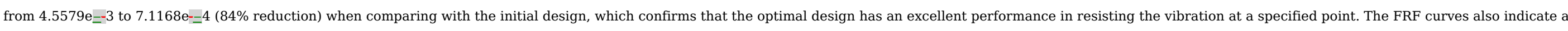

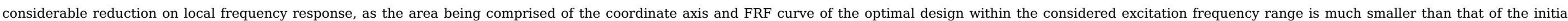
design. 


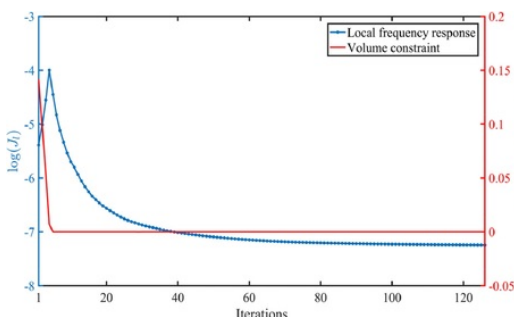

(a)

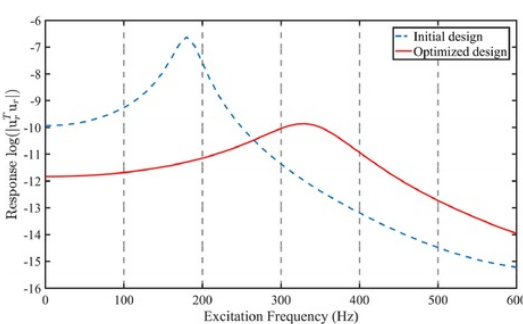

(b)

Fig. 5 Curves of first example using iPLSM with $\mathrm{k}=1$ : (a) convergent histories of objective function and volume constraint; (b) FRF of excitation point before and after optimization.

\section{alt-text: Fig. 5.}

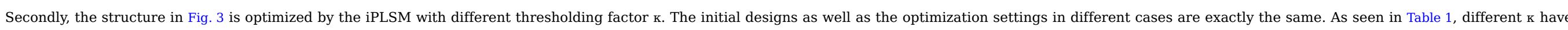

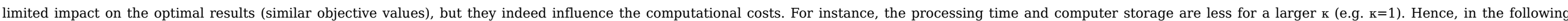
examples, $\mathrm{k}=1$ will be adopted.

Table 1 Investigation of iPLSM with different $\mathrm{K}$.

alt-text: Table 1

\begin{tabular}{|c|c|c|c|c|c|}
\hline $\mathrm{K}$ & Size of matrix $\mathbf{A}$ & Sparsity of matrix $\widetilde{\mathbf{A}}_{s}$ & Time per step & Iterations & Objective \\
\hline $1 e-500$ & $11,521 \times 11,521$ & $64.27 \%$ & 128.1_s & 124 & $7.1202 \mathrm{e}_{-}^{-}=4$ \\
\hline $1 \mathrm{e}_{-}^{-}=300$ & $11,521 \underline{521} *-\underline{x} 11_{2} 521$ & $86.13 \%$ & 69.1_s & 125 & $7.1170 \mathrm{e}_{-}^{-}=4$ \\
\hline $1 \mathrm{e}-=200$ & $11_{2} 521 \underline{521} *-\underline{x} 11_{2} 521$ & $89.94 \%$ & $68.2 \_s$ & 125 & $7.1169 \mathrm{e}-=4$ \\
\hline 1 & $11_{2} 521 \underline{521} *-\underline{x} 11_{2} 521$ & $99.74 \%$ & 65.3_s & 125 & $7.1168 \mathrm{e}-=4$ \\
\hline
\end{tabular}

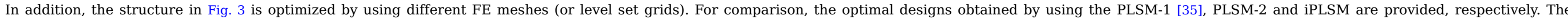

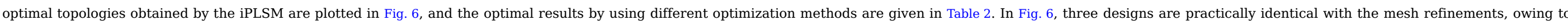
the filter scheme in the proposed method. It confirms that the iPLSM can alleviate the mesh-dependency in the optimization.

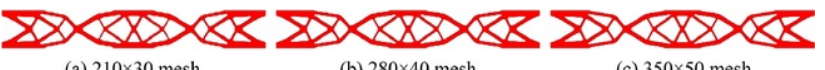

$\begin{array}{lll}\text { (a) } 210 \times 30 \text { mesh } & \text { (b) } 280 \times 40 \text { mesh } & \text { (c) } 350 \times 50 \text { mesh }\end{array}$

alt-text: Fig. 6.

Table 2 Comparison of different methods in local frequency response optimization.

alt-text: Table 2

\begin{tabular}{|c|c|c|c|c|c|c|}
\hline Method & Mesh & Size of matrix $\mathbf{A}$ & Sparsity of matrix $\mathbf{A}$ or $\widetilde{\mathbf{A}}_{s}$ & Time per step & Iterations & Objective \\
\hline iPLSM & $210-\underline{210} *-\underline{x} 30$ & $6541 \underline{6541} *-\underline{x} 6541$ & $99.54 \%$ & $24.3 \_s$ & 119 & $7.0187 \mathrm{e}^{-}=4$ \\
\hline PLSM-1 & $210 \underline{210} *-\underline{x} 30$ & $6541 \underline{6541} *-\underline{x} 6541$ & $99.18 \%$ & 24.6_s & 145 & $7.3016 \mathrm{e}_{-}^{-}=4$ \\
\hline PLSM-2 & $210 \underline{210} *-\underline{x} 30$ & $6541 \underline{6541} *-\underline{x} 6541$ & $0 \%$ & 64.6_s & 105 & $7.0443 \mathrm{e}=4$ \\
\hline iPLSM & $280-280 *-\times 40$ & $11,521-521 *-\times 11,521$ & $99.74 \%$ & $65.3 \mathrm{~s}$ & 125 & $7.1168 \mathrm{e}--4$ \\
\hline
\end{tabular}




\begin{tabular}{|c|c|c|c|c|c|c|}
\hline PLSM-1 & $280 \underline{280} *-\underline{x} 40$ & $11_{2} 521 \underline{521} *-\underline{x} 11_{2} 521$ & $99.53 \%$ & 65.9_s & 161 & $7.3518 \mathrm{e}-=4$ \\
\hline PLSM-2 & $280 \underline{280} * \underline{x} 40$ & $11_{2} 521 \underline{521} * \underline{x} 11_{2} 521$ & $0 \%$ & 197.2_s & 127 & $7.1105 \mathrm{e}-=4$ \\
\hline iPLSM & $350 \underline{350} *-\underline{x} 50$ & $17_{\alpha}, 901 \underline{901} *-\underline{x} 17_{2} 901$ & $99.83 \%$ & 128.6_s & 136 & $7.1280 \mathrm{e}=4$ \\
\hline PLSM-1 & $350 \underline{350} *-\underline{x} 50$ & $17,901 \underline{901} *-\underline{x} 17_{2} 901$ & $99.69 \%$ & 129.5_s & 202 & $7.4264 \mathrm{e}_{-}=4$ \\
\hline PLSM-2 & $350-\underline{350} *-\underline{x} 50$ & $17,901 \underline{901} *-\underline{x} 17,901$ & $0 \%$ & 459.7_s & 134 & $7.1344 \mathrm{e}_{-}^{-}=4$ \\
\hline
\end{tabular}

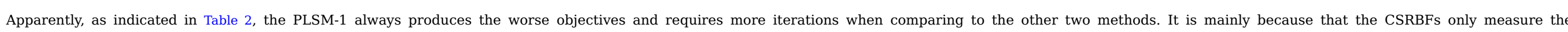

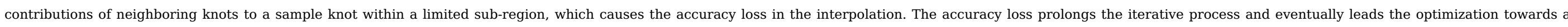

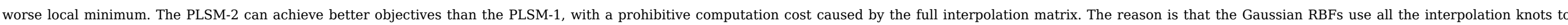

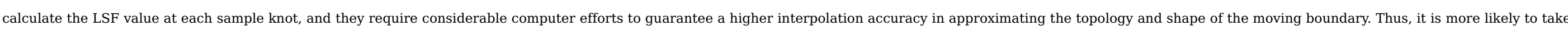
fewer iterations to find an optimized design with higher performance.

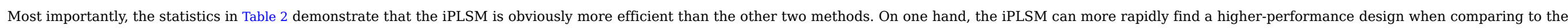

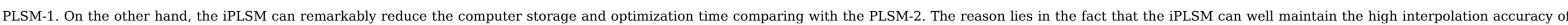
the GSRBF, while efficiently compress the full interpolation matrix by using the DWT.

\subsection{Local frequency response optimization for multiple points of $2 \mathrm{D}$ structure}

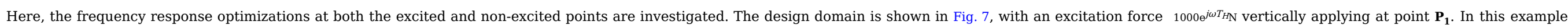

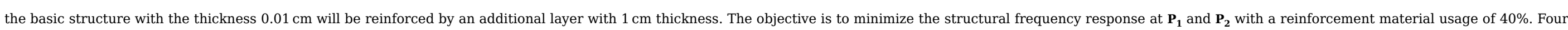

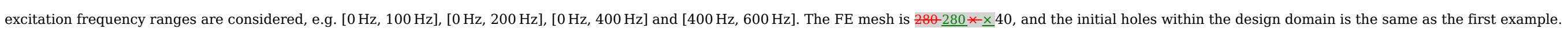

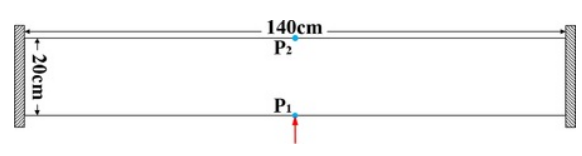

Fig. 7 Design domain of second example.

alt-text: Fig. 7.

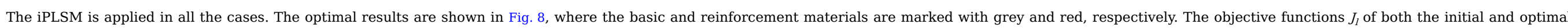

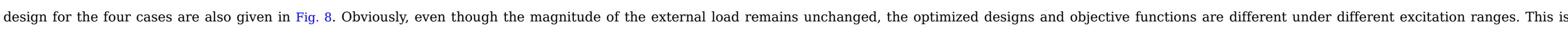

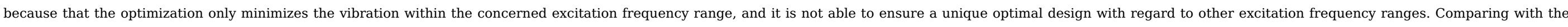

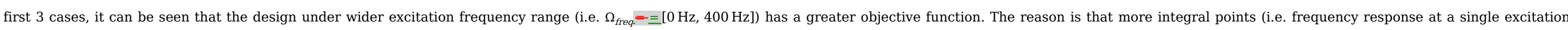

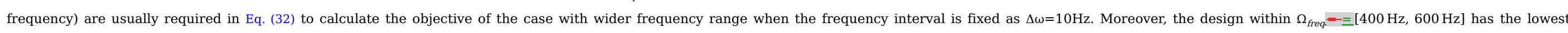
objective among the 4 cases. It implies that higher frequency range (i.e. [400 Hz, $600 \mathrm{~Hz}]$ ) may have a lower frequency response in this problem. 


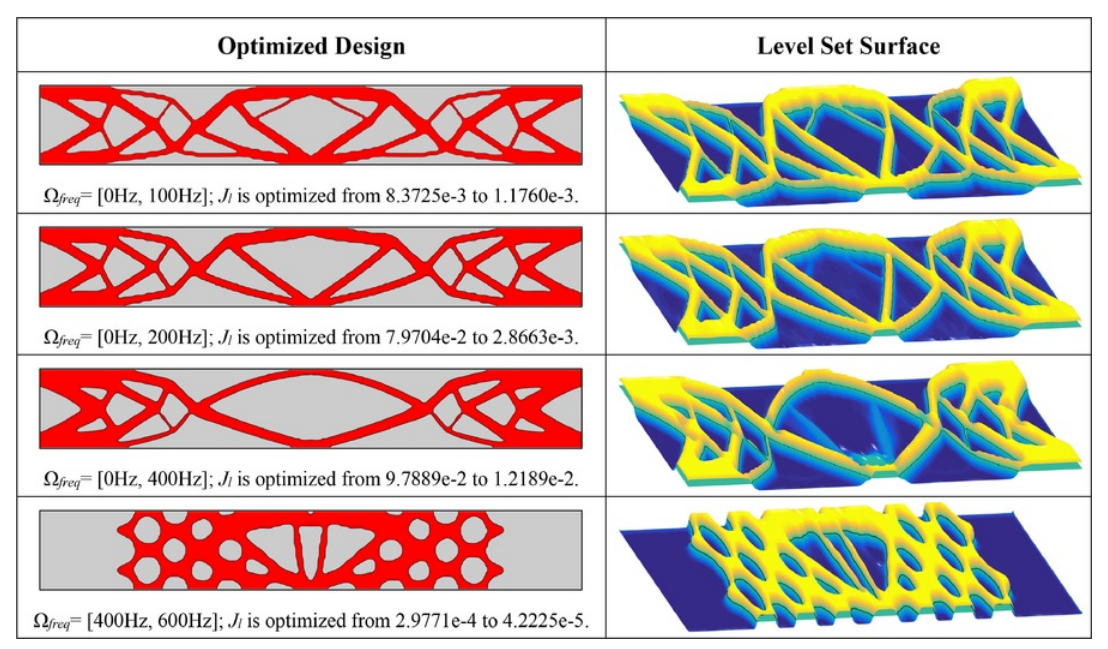

Fig. 8 Optimized designs of second example under different excitation frequency ranges.

\section{alt-text: Fig. 8.}

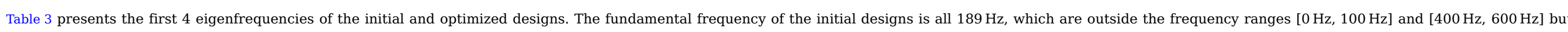

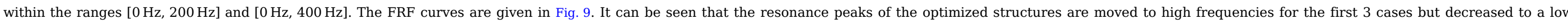

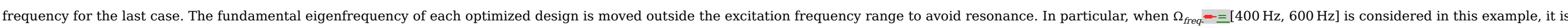

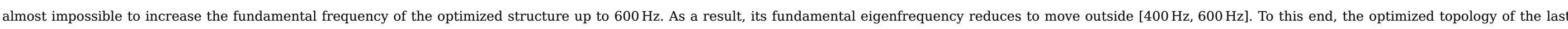

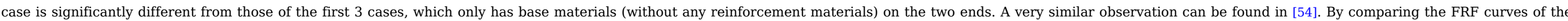

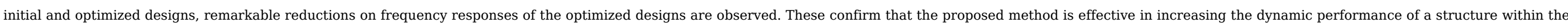
concerned excitation frequency range.

Table 3 Eigenfrequencies of initial design and optimized designs.

alt-text: Table 3

Eigenfrequency order

Initial design

Optimized design with $\Omega_{\text {freq }}=\equiv[0 \mathrm{~Hz}, 100 \mathrm{~Hz}]$

Optimized design with $\Omega_{\text {freq }}=\equiv[0 \mathrm{~Hz}, 200 \mathrm{~Hz}]$

Optimized design with $\Omega_{\text {freq }}==[0 \mathrm{~Hz}, 400 \mathrm{~Hz}]$

Optimized design with $\Omega_{\text {freq }}=\equiv[400 \mathrm{~Hz}, 600 \mathrm{~Hz}]$

\section{1 st}

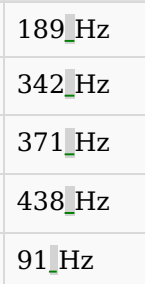

\begin{abstract}
2nd
\end{abstract}

484_Hz

564_Hz

564_Hz

577_Hz

203_Hz

\section{3rd}

739_Hz

853_Hz

878_Hz

1000_Hz

210_Hz 4th

869_Hz

1085_Hz

1085_Hz

1141_Hz

717_Hz 


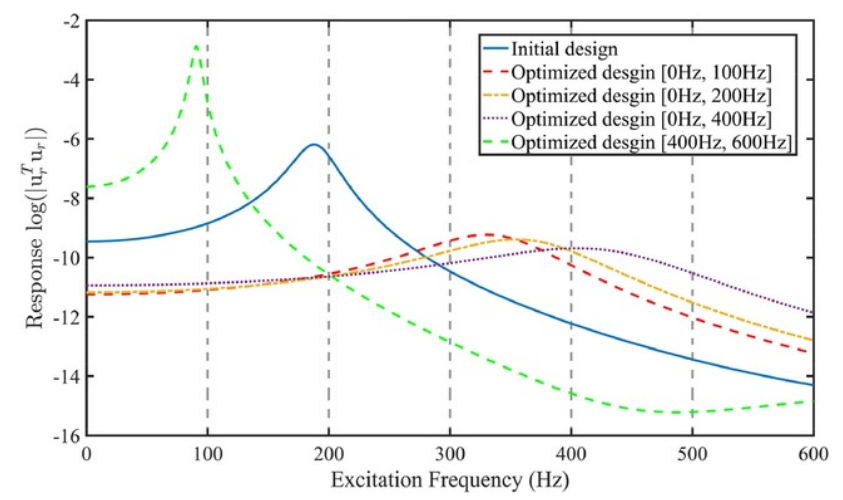

Fig. 9 FRF of points $\mathbf{P}_{\mathbf{1}}$ and $\mathbf{P}_{\mathbf{2}}$ before and after optimization. (For interpretation of the references to color in this figure legend, the reader is referred to the web version of this article.)

\section{alt-text: Fig. 9.}

\subsection{Global frequency response optimization of 2D structure}

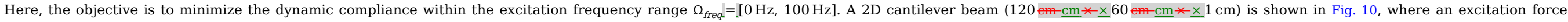
$100 \mathrm{e}^{j \omega T_{H N}}$ is vertically applied at point $\mathbf{P}$. The FE mesh is $120 \underline{120} * \underline{x} 60$. Different volume constraints are examined, namely $50 \%, 40 \%$ and $30 \%$.

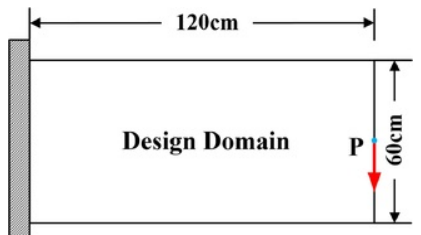

Fig. 10 Design domain of third example.

alt-text: Fig. 10.

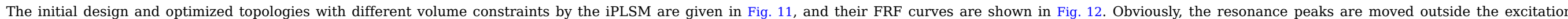

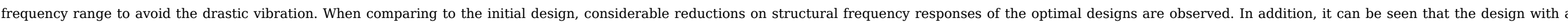

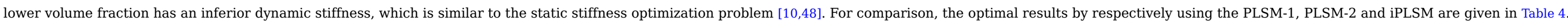

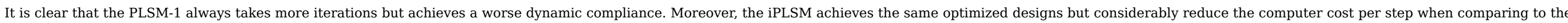

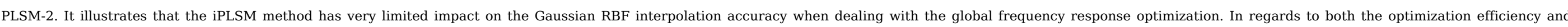
effectiveness, the iPLSM performs best amongst the three methods. 


\section{8}

(a) Initial design

(b) Optimized design with $50 \%$ volume

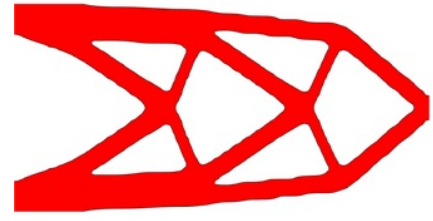

(c) Optimized design with $40 \%$ volume

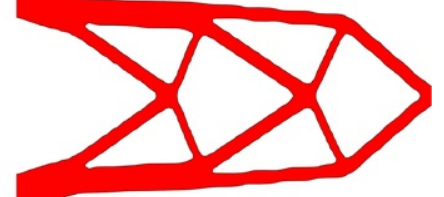

(d) Optimized design with $30 \%$ volume

Fig. 11 Initial design and optimized designs of third example under different volume constraints.

alt-text: Fig. 11.

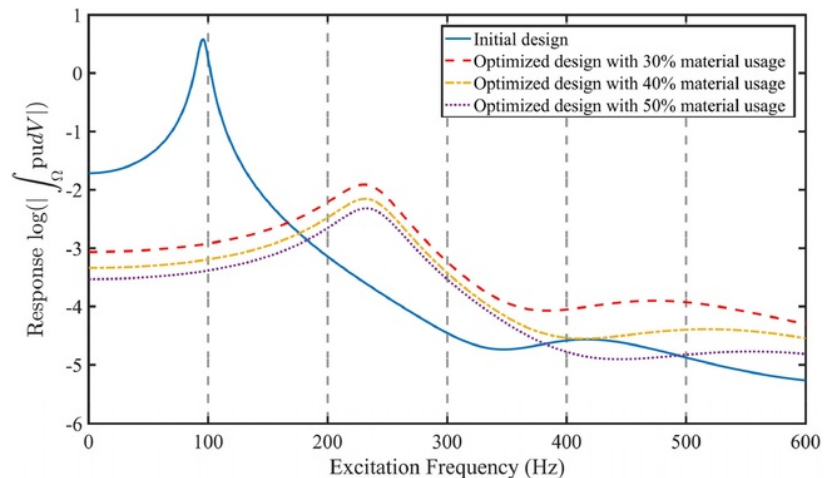

Fig. 12 FRF of initial design and optimized designs.

\section{alt-text: Fig. 12.}

Table 4 Comparison of different methods in 2D global frequency response optimization.

alt-text: Table 4

\begin{tabular}{|c|c|c|c|c|c|}
\hline Method & Sparsity of matrix $\mathbf{A}$ or $\tilde{\mathbf{A}}_{s}$ & Volume constraint & Time per step & Iterations & Objective \\
\hline iPLSM & $99.59 \%$ & $50 \%$ & $26.3 \_s$ & 112 & 1.1863 \\
\hline PLSM-1 & $99.26 \%$ & $50 \%$ & $26.5 \_s$ & 165 & 1.2081 \\
\hline PLSM-2 & $0 \%$ & $50 \%$ & $69.8 \_s$ & 112 & 1.1863 \\
\hline iPLSM & $99.59 \%$ & $40 \%$ & $26.2 \_s$ & 160 & 1.4370 \\
\hline PLSM-1 & $99.26 \%$ & $40 \%$ & $26.5 \_s$ & 220 & 1.4926 \\
\hline PLSM-2 & $0 \%$ & $40 \%$ & $69.9 \_s$ & 160 & 1.4370 \\
\hline iPLSM & $99.59 \%$ & $30 \%$ & $26.3 \_s$ & 176 & 1.8928 \\
\hline PLSM-1 & $99.26 \%$ & $30 \%$ & $26.4 \_s$ & 260 & 1.9822 \\
\hline
\end{tabular}




\subsection{Local frequency response optimization of 3D structure}

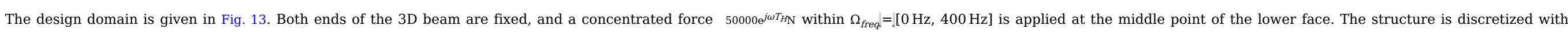

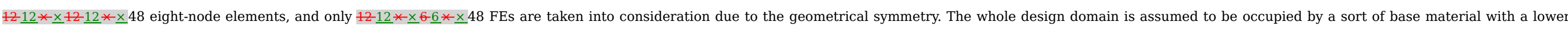

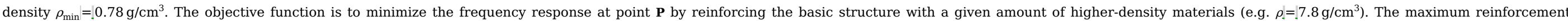
material usage is $30 \%$.

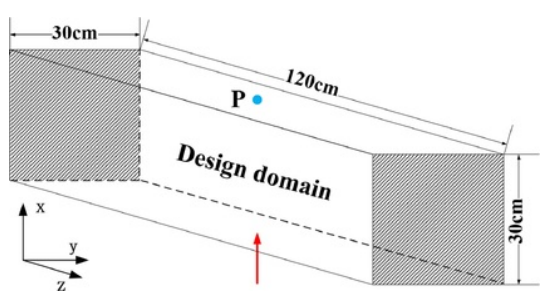

Fig. 13 Design domain of fourth example.

alt-text: Fig. 13.

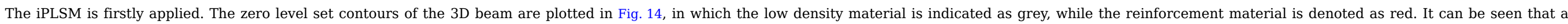

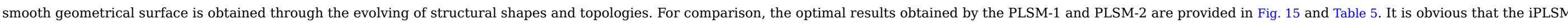

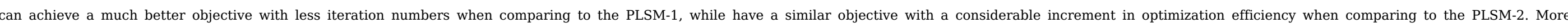

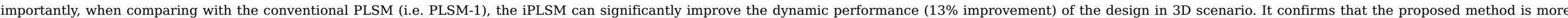
efficient and effective in dealing with 3D optimization design problem.
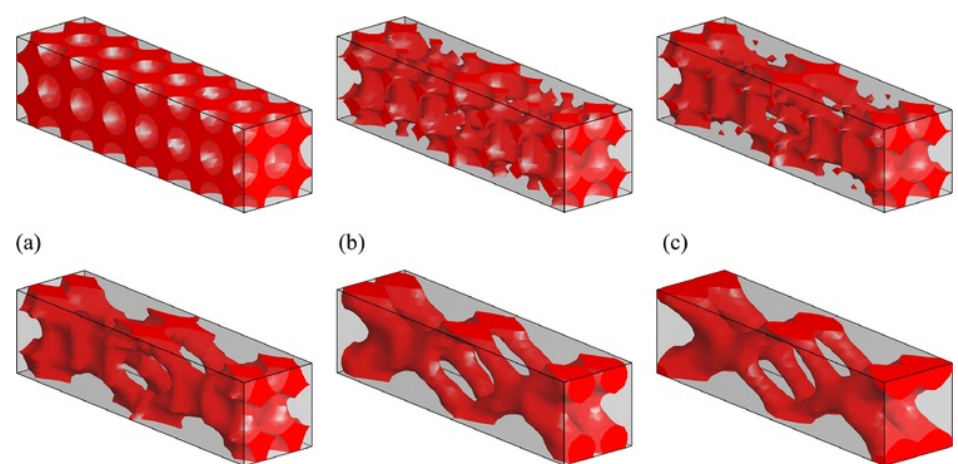

(b)

(c)
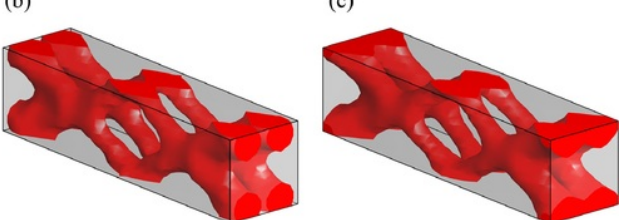

Fig. 14 Evolution of structural boundary of fourth example: (a) initial design; (b) step 15; (c) step 20; (d) step 25; (e) step 50; (f) optimized design.

alt-text: Fig. 14. 


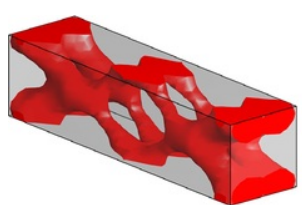

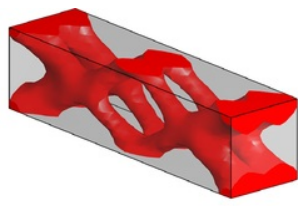

Fig. 15 Optimal designs of fourth example: (a) PLSM-1; (b) PLSM-2.

alt-text: Fig. 15.

Table 5 Comparison of different methods in fourth example. alt-text: Table 5

\begin{tabular}{|c|c|c|c|c|c|c|}
\hline Method & Mesh & Size of matrix $\mathbf{A}$ & Sparsity of matrix $\mathbf{A}$ or $\widetilde{\mathbf{A}}_{s}$ & Time per step & Iterations & Objective \\
\hline iPLSM & $12 \underline{12} *-\underline{x} 6 \underline{6} *-\underline{x} 48$ & $4459 \underline{4459} *-\underline{x} 4459$ & $96.15 \%$ & 236.3_s & 96 & $2.1872 \mathrm{e}_{-}=3$ \\
\hline PLSM-1 & $12 \underline{12} *-\underline{x} 6 \underline{6} *-\underline{x} 48$ & $4459 \underline{4459} *-\underline{x} 4459$ & $89.06 \%$ & $241.6 \_s$ & 108 & $2.5075 \mathrm{e}-=3$ \\
\hline PLSM-2 & $12 \underline{12} \times-\underline{x} 6 \underline{6} \times-\underline{x} 48$ & $4459-\underline{4459} *-\underline{x} 4459$ & $0 \%$ & 269.6_s & 96 & $2.1864 \mathrm{e}=3$ \\
\hline
\end{tabular}

\subsection{Global frequency response optimization of 3D structure}

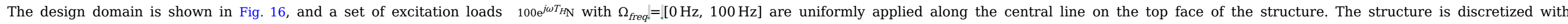

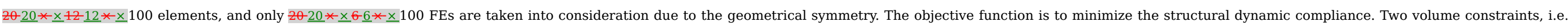
$40 \%$ and $20 \%$, are considered.

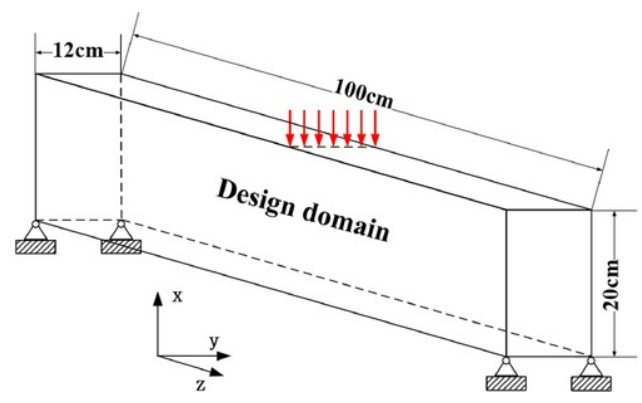

Fig. 16 Design domain of fifth example.

\section{alt-text: Fig. 16.}

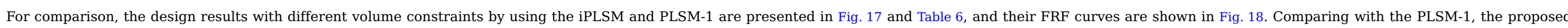

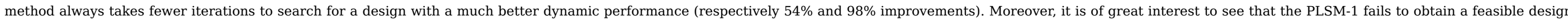

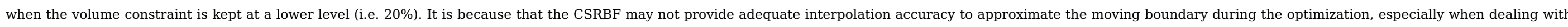

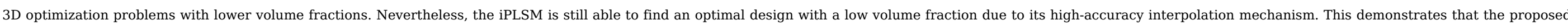
method is more efficient and effective than the conventional PLSM. 

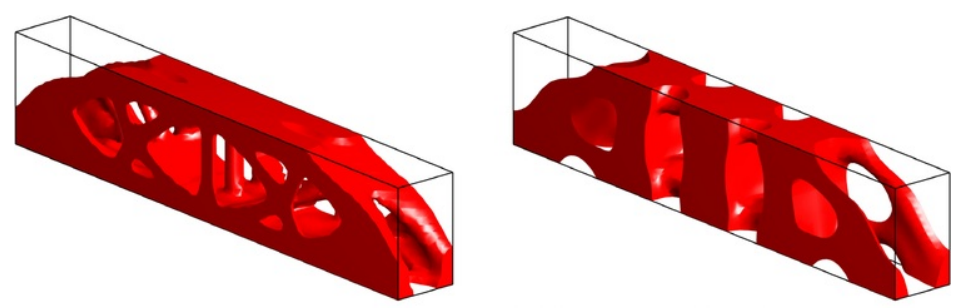

(a) Designs with 40\% material usage: iPLSM (Left); PLSM-1 (Right)

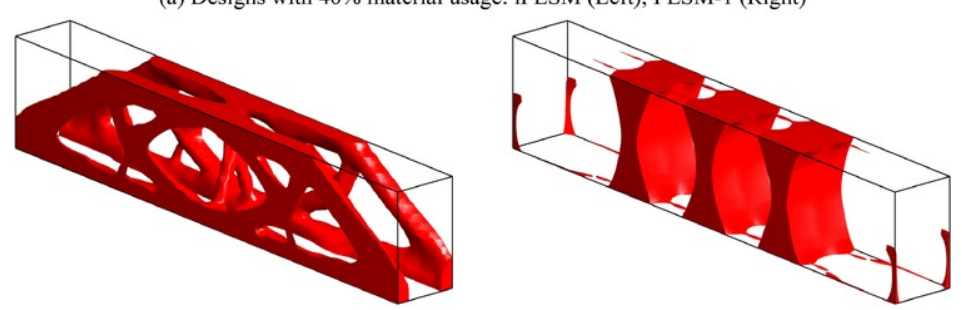

(b) Designs with 20\% material usage: iPLSM (Left); PLSM-1 (Right)

Fig. 17 Design results of fifth example using different methods.

\section{alt-text: Fig. 17.}

Table 6 Comparison of different methods in fifth example.

alt-text: Table 6

\begin{tabular}{|c|c|c|c|c|c|c|}
\hline Method & Size of matrix $\mathbf{A}$ & Sparsity of matrix $\mathbf{A}$ or $\widetilde{\mathbf{A}}_{s}$ & Volume & Time per setp & Iterations & Objective \\
\hline iPLSM & $14,847 \underline{847} *-\underline{x} 14,847$ & $99.38 \%$ & $40 \%$ & $585.6 \_s$ & 116 & 34.7718 \\
\hline PLSM-1 & $14,847 \underline{847} *-\underline{x} 14,847$ & $96.34 \%$ & $40 \%$ & $592.2 \_\mathrm{s}$ & 400 & 75.8112 \\
\hline iPLSM & $14,847 \underline{847} *-\underline{x} 14_{2} 847$ & $99.38 \%$ & $20 \%$ & $585.8 \_s$ & 142 & 59.1339 \\
\hline PLSM-1 & $14,847 \underline{847} *-\underline{x} 14,847$ & $96.34 \%$ & $20 \%$ & 592.4_s & 215 & 2703.0524 \\
\hline
\end{tabular}

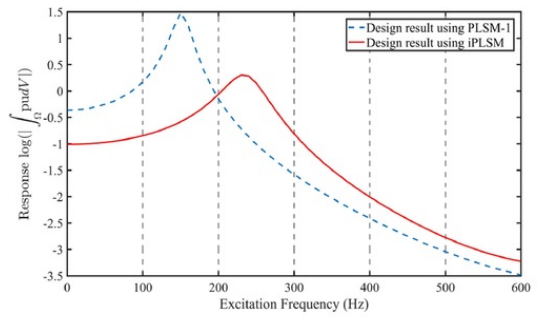

(a)

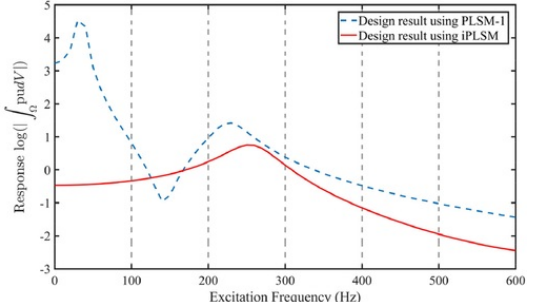

Fig. 18 FRF curves of optimized designs: (a) volume constraint is $40 \%$; (b) volume constraint is $20 \%$. alt-text: Fig. 18.

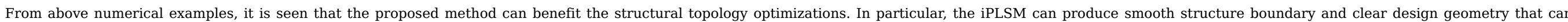

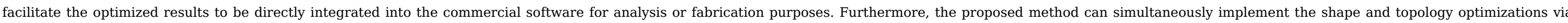

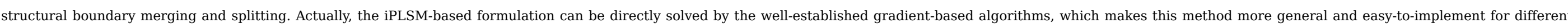




\section{Conclusions}

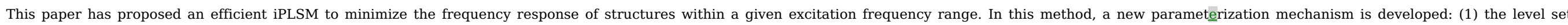

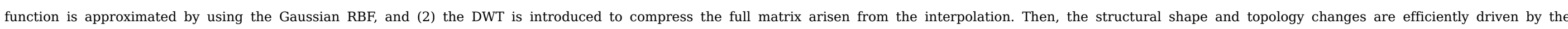

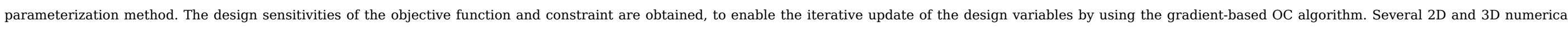

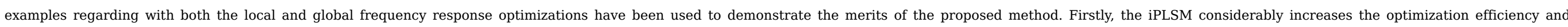

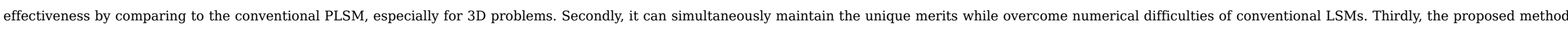

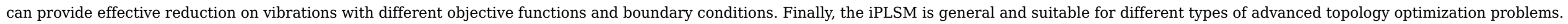

\section{Acknowledgements}

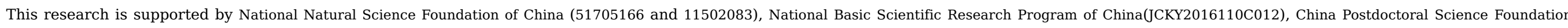

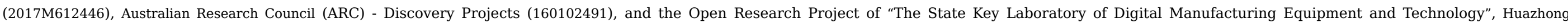
University of Science and Technology (DMETKF2015010), Wuhan, China.

\section{References}

[1] AGM Michell, The limit of economy of material in frame structures, Philos Mag 8, 1904, 589-597.

[2] BHV Topping, Shape optimization of skeletal structures: Aa review, J Struct Eng 109, 1983, 1933-1951.

[3] HL Cox, The design of structures of least weight, 1965, Pergamon Press; Oxford.

[4] W Hemp and HSY Chan, Optimum design of pin-jointed frameworks, 1966, Her Majesty's Stationary Office, London: R. \& M. 3632.

[5] W Hemp, Optimum structures, 1973, Clarendon Press; Oxford.

[6] HSY Chan, The design of michell optimum structures, 1960, Cranfield: College of Aeronautics, Technical report.

[7] W Prager and GIN Rozvany, Optimal layout of grillages, J Struct Mech 5, 1977, 1-18.

[8] GD Cheng and N Olhoff, An investigation concerning optimal design of solid elastic plates, Int J Solids Struct 17, 1981, 305-323.

[9] GD Cheng and N Olhoff, Regularized formulation for optimal design of axisymmetric plates, Int J Solids Struct 18, 1982, 153-169.

[10] MP Bendsøe and O Sigmund, Topology optimization: theory, methods, and applications, 2003, Springer; Berlin, Heidelberg.

[11] O Sigmund and K Maute, Topology optimization approaches: a comparative review, Struct Multidiscip Optim 48, 2013, $1031-1055$.

[12] MP Bendsøe and N Kikuchi, Generating optimal topologies in structural design using a homogenization method, Comput Methods Appl Mech Engrg 71, 1988, 197-224.

[13] M Zhou and GIN Rozvany, The COC algorithm, part II: Ғtopological, geometry and generalized shape optimization, Comput Methods Appl Mech Eng 89, 1991, 309-336.

[14] MP Bendsøe and O Sigmund, Material interpolation schemes in topology optimization, Arch Appl Mech 69, 1999, 635-654.

[15] YM Xie and GP Steven, A simple evolutionary procedure for structural optimization, Comput Struct 49, 1993, 885-896.

[16] S Osher and JA Sethian, Fronts propagating with curvature-dependent speed-algorithms based on Hamilton-Jacobi formulations, J Comput Phys 79, $1988,12-49$.

[17] JA Sethian and A Wiegmann, Structural boundary design via level set and immersed interface methods, J Comput Phys 163, 2000, 489-528. 
[18] S Osher and RP Fedkiw, Level set methods and dynamic implicit surface, 2002, Springer; New York.

[19] MY Wang, XM Wang and DM Guo, A level set method for structural topology optimization, Comput Methods Appl Mech Engæg 192, 2003, 227-246.

[20] G Allaire, F Jouve and AM Toader, Structural optimization using sensitivity analysis and a level-set method, J Comput Phys 194, 2004, 363-393.

[21] NP van Dijk, K Maute, M Langelaar and F van Keulen, Level-set methods for structural topology optimization: a review, Struct Multidiscip Optim 48, 2013 , 437-472.

[22] G Allaire and F Jouve, A level-set method for vibration and multiple loads structural optimization, Comput Methods Appl Mech Engғg 194, 2004, 3269-3290.

[23] MY Wang and XM Wang, "Color" level sets: a multi-phase method for structural topology optimization with multiple materials, Comput Methods Appl Mech Engғg 193, 2004, 469-496.

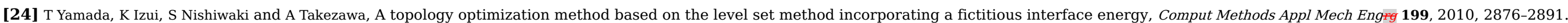

[25] SW Zhou, W Li, GY Sun and Q Li, A level-set procedure for the design of electromagnetic metamaterials, Opt Express 18, 2010, 6693-6702.

[26] SK Chen and W Chen, A new level-set based approach to shape and topology optimization under geometric uncertainty, Struct Multidiscip Optim 44, 2011 , 1-18.

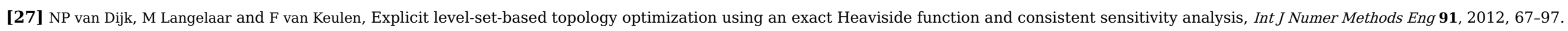

[28] X Guo, W Zhang and W Zhong, Explicit feature control in structural topology optimization via level set method, Comput Methods Appl Mech Engrg 272, 2014, 354-378.

[29] PD Dunning and HA Kim, Introducing the sequential linear programming level-set method for topology optimization, Struct Multidiscip Optim 51, 2015 , 631-643.

[30] K. Svanberg, The method of moving asymptotes: a new method for structural optimization, Int J Numer Methods Eng 24, 1987, 359-373.

[31] T Belytschko, SP Xiao and C Parimi, Topology optimization with implicitly function and regularization, Int J Numer Methods Eng 57, 2003, 1177-1196.

[32] Q Xia, MY Wang, SY Wang and SK Chen, Semi-Lagrange method for level-set-based structural topology and shape optimization, Struct Multidiscip Optim 31, 2006 , 419-429.

[33] JZ Luo, Z Luo, LY Tong and MY Wang, A semi-implicit level set method for structural shape and topology optimization, J Comput Phys 227, 2008 , 5561-5581.

[34] MD Zhou and MY Wang, A semi-Lagrangian level set method for structural optimization, Struct Multidiscip Optim 46, 2012 , 487-501.

[35] Z Luo, MY Wang, SY Wang and P Wei, A level set-based parameterization method for structural shape and topology optimization, Int J Numer Methods Eng 76, 2008 , 1-26.

[36] Z Luo, LY Tong and Z Kang, A level set method for structural shape and topology optimization using radial basis functions, Comput Struct 87, 2009, 425-434.

[37] YQ Wang, Z Luo, N Zhang and Z Kang, Topological shape optimization of microstructural metamaterials using a level set method, Comput Mater Sci 87, 2014, 178-186.

[38] MD Buhmann, Radial basis functions: theory and implementations, Cambridge monographs on applied and computational mathematics 12, 2004, Cambridge University Press; New York.

[39] H Wendland, Computational aspects of radial basis function approximation, 2005, Elsevier BV.

[40] J Ravnik, L Škerget and M Hriberšek, The wavelet transform for BEM computational fluid dynamics, Eng Anal Bound Elem 28, 2004, $1303-1314$.

[41] K Chen, Discrete wavelet transforms accelerated sparse preconditioners for dense boundary element systems, Electron Trans Numer Anal 8, 1999, 138-153.

[42] NL Pedersen, Maximization of eigenvalues using topology optimization, Struct Multidiscip Optim 20, 2000, 2-11.

[43] TS Kim and YY Kim, MAC-based mode-tracking in structural topology optimization, Comput Struct 74, 2000, 375-383.

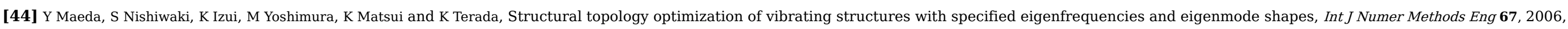
597-628. 


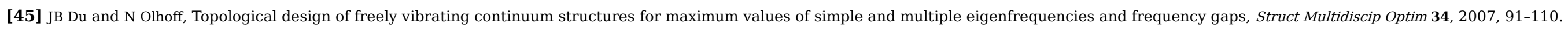

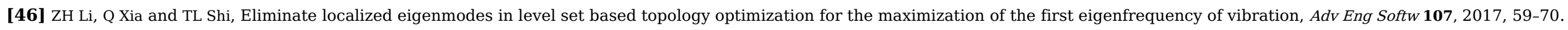

[47] ZD Ma, N Kikuchi and I Hagiwara, Structural topology and shape optimization for a frequency response problem, Comput Mech 13, 1993, 157-174.

[48] ZD Ma, N Kikuchi and HC Cheng, Topological design for vibrating structures, Comput Methods Appl Mech Engrg 121, 1995, $259-280$.

[49] CS Jog, Topology design of structures subjected to periodic loading, J Sound Vibæ 253, 2002, 687-709.

[50] D Tcherniak, Topology optimization of resonating structures using SIMP method, Int J Numer Methods Eng 54, 2002, 1605-1622.

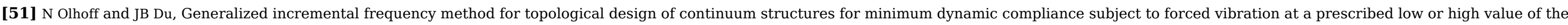
excitation frequency, Struct Multidiscip Optim 54, 2016, 1113-1141.

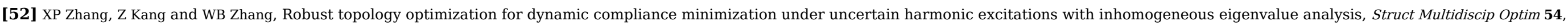
2016, 1469-1484.

[53] JS Jensen, Topology optimization of dynamics problems with Padé approximants, Int J Numer Methods Eng 72, 2007, 1605-1630.

[54] GH Yoon, Structural topology optimization for frequency response problem using model reduction schemes, Comput Methods Appl Mech Engrg 199, $2010,1744-1763$.

[55] L Shu, MY Wang, ZD Fang, ZD Ma and P Wei, Level set based structural topology optimization for minimizing frequency response, J Sound Vibæ 330, 2011, 5820-5834.

[56] JH Rong, ZL Tang, YM Xie and FY Li, Topological optimization design of structures under random excitations using SQP method, Eng Struct 56, 2013, 2098-2106.

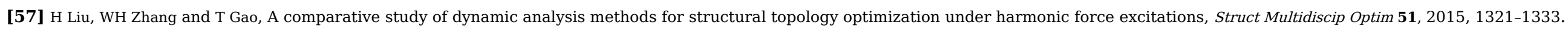

[58] G Beylkin, R Coifman and V Rokhlin, Fast wavelet transforms and numerical algorithms, Commun Pure Appl Math 44, 1991, 141-183.

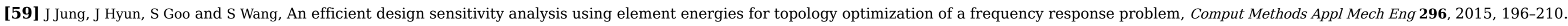

[60] KK Choi and NH Kim, Structural sensitivity analysis and optimization - linear systems, 2005, Springer; New York.

[61] R Ohayon and C Soize, Structural acoustics and vibration, 1998, Academic Press; New York.

[62] XP Zhang and Z Kang, Topology optimization of piezoelectric layers in plates with active vibration control, J Intell Mater Syst Struct 25, 2014, 697-712.

[63] J Sokolowski and JP Zolesio, Introduction to shape optimization: shape sensitivity analysis, 1992, Springer-Verlag; New York.

[64] M Bruyneel and P Duysinx, Note on topology optimization of continuum structures including self-weight, Struct Multidiscip Optim 29, $2005,245-256$.

[65] JH Zhu, P Beckers and WH Zhang, On the multi-component layout design with inertial force, J Comput Appl Math 234, 2010, 2222-2230.

[66] T Belytschko, C Parimi, N Moes, N Sukumar and S Usui, Structured extended finite element methods for solids defined by implicit surfaces, Int J Numer Methods Eng 56, 2002 , 609-635.

[67] B Bourdin, Filters in topology optimization, Int J Numer Methods Eng 50, 2001, 2143-2158.

[68] O Sigmund, A 99 line topology optimization code written in Matlab, Struct Multidiscip Optim 21, 2001, 120-127.

[69] GH Yoon, Toward a multifrequency quasi-static Ritz vector method for frequency-dependent acoustic system application, Int J Numer Methods Eng 89, $2012,1451-1470$. 
Highlights

- An improved parametric level set method is proposed.

- The discrete wavelet transform is used to compress the Gaussian RBF-based interpolation matrix.

- Both self and non-self adjoint problems for global and local frequency responses are investigated.

- The efficiency and effectiveness of the proposed method are verified.

\section{Queries and Answers}

Query: Please confirm that givennames and surnames have been identified correctly.

Answer: Yes

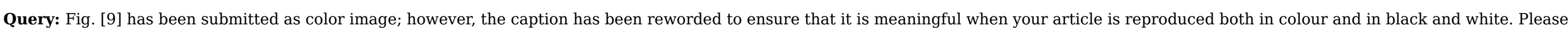
check and correct if necessary.

Answer: yes, thanks

Query: Please check funding information and confirm its correctness.

Answer: yes, thanks

Query: Please check funding information and confirm its correctness.

Answer: yes, thanks 